\title{
A New Strategy for the Total Synthesis of Macrosphelide A and B Based on the Ring-Closing Metathesis
}

\author{
Yuji Matsuya, Takanori Kawaguchi, and Hideo Nemoto* \\ Faculty of Pharmaceutical Sciences, Toyama Medical and Pharmaceutical University, \\ Sugitani 2630, Toyama 930-0194, Japan \\ Fax : +81-76-434-5047 \\ E-mail :nemotoh@ms.toyama-mpu.ac.jp
}

\section{Supporting Information}

Experimental procedures and characterization data for all new compounds.

General Procedures. All nonaqueous reactions were carried out under Ar atmosphere. Reagents were purchased from commercial sources and used as received. Methyl $(S)$-()-lactate (optically pure reagent) and methyl $(S)-(+)-3$-hydroxybutyrate $(>80 \%$ ee) were purchased from Tokyo Kasei Kogyo Co., Ltd and Wako Pure Chemical Industries, Ltd, respectively. Anhydrous solvents were obtained from commercial sources or prepared by distillation over $\mathrm{CaH}_{2}$ or $\mathrm{P}_{2} \mathrm{O}_{5} .{ }^{1} \mathrm{H}$ and ${ }^{13} \mathrm{C}$ NMR spectra were obtained on a Varian Gemini $300\left(300 \mathrm{MHz}\right.$ for ${ }^{1} \mathrm{H}$ and $75.46 \mathrm{MHz}$ for ${ }^{13} \mathrm{C}$ ) instrument or a Varian UNITY plus $500\left(500 \mathrm{MHz}\right.$ for ${ }^{1} \mathrm{H}$ and $125 \mathrm{MHz}$ for ${ }^{13} \mathrm{C}$ ) instrument, using tetramethylsilane or chloroform as an internal reference. Mass spectra were measured on a JEOL D-200 or a JEOL AX 505 mass spectrometer, and the ionization method was electron impact (EI, $70 \mathrm{eV}$ ). IR spectra were recorded on a Perkin-Elmer 1600 spectrometer. The optical rotations were determined on a JASCO DIP-1000 instrument. Melting points were taken with a Yanagimoto micro melting point apparatus and are uncorrected. Column chromatography was carried out by employing Cica Silica Gel 60N (spherical, neutral, 
$40-50 \mu \mathrm{m}$ or $63-210 \mu \mathrm{m})$.

$(3 R, 4 S)-4-($ tert-Butyldimethylsilyloxy)-3-[(2-methoxyethoxy)methoxy]-1-pentene

(9). (2-Methoxyethoxy)methyl chloride $(7.1 \mathrm{ml}, 63 \mathrm{mmol})$ was added dropwise to a stirred solution of the alcohol $7^{5)}(6.8 \mathrm{~g}, 21 \mathrm{mmol})$ and $N, N$-diisopropylethylamine $(16.5$ $\mathrm{ml}, 95 \mathrm{mmol})$ in $\mathrm{CH}_{2} \mathrm{Cl}_{2}(100 \mathrm{ml})$ at $0{ }^{\circ} \mathrm{C}$ under Ar atmosphere. After continuous stirring for $24 \mathrm{~h}$ at room temperature, the reaction mixture was diluted with $\mathrm{CH}_{2} \mathrm{Cl}_{2}(25$ $\mathrm{ml}$ ), washed with water, $10 \% \mathrm{HCl}$, sat. $\mathrm{NaHCO}_{3}$, and brine successively, and dried over $\mathrm{MgSO}_{4}$. Evaporation of the solvent afforded a residue which was chromatographed on silica gel with hexane-EtOAc $(10: 1)$ to give MEM ether $9(6.71 \mathrm{~g}, 70 \%)$ as a colorless oil. ${ }^{1} \mathrm{H}$ NMR (300 MHz, $\left.\mathrm{CDCl}_{3}\right): \delta 5.71(1 \mathrm{H}, \mathrm{ddd}, J=17,10,7.4 \mathrm{~Hz}), 5.25(1 \mathrm{H}, \mathrm{d}, J=10$ $\mathrm{Hz}), 5.24(1 \mathrm{H}, \mathrm{d}, J=17 \mathrm{~Hz}), 4.77(1 \mathrm{H}, \mathrm{d}, J=6.8 \mathrm{~Hz}), 4.68(1 \mathrm{H}, \mathrm{d}, J=6.8 \mathrm{~Hz}), 3.89-3.77$ $(3 \mathrm{H}, \mathrm{m}), 3.66-3.51(3 \mathrm{H}, \mathrm{m}), 3.39(3 \mathrm{H}, \mathrm{s}), 1.14(3 \mathrm{H}, \mathrm{d}, J=6.0 \mathrm{~Hz}), 0.88(9 \mathrm{H}, \mathrm{s}), 0.05(3 \mathrm{H}$, s), $0.04(3 \mathrm{H}, \mathrm{s}) ;{ }^{13} \mathrm{C}$ NMR $\left(75 \mathrm{MHz}, \mathrm{CDCl}_{3}\right): \delta 135.15,118.53,92.74,81.48,71.60$, 70.51, 66.70, 58.82, 25.76, 19.58, 18.04, -4.64, -4.67; IR (neat): $1644 \mathrm{~cm}^{-1}$; MS (EI) $\mathrm{m} / \mathrm{z}$ $304\left(\mathrm{M}^{+}\right)$; HRMS (EI) Calcd for $\mathrm{C}_{15} \mathrm{H}_{32} \mathrm{O}_{4} \mathrm{Si}$ : $304.2070\left(\mathrm{M}^{+}\right)$, found: 304.2092 ; $[\alpha]_{\mathrm{D}}{ }^{25}$ $-56.8\left(c=1.22, \mathrm{CHCl}_{3}\right)$.

(2S,3S)-3-(tert-Butyldimethylsilyloxy)-2-[(2-methoxyethoxy)methoxy]butanal (10). Osmium tetroxide (catalytic amount) and $50 \%$ aqueous $N$-methylmorpholine $N$-oxide $(6.15 \mathrm{ml}, 26 \mathrm{mmol})$ were added to a solution of MEM ether 9 (4.00 g, $13 \mathrm{mmol})$ in acetone $(60 \mathrm{ml})$ at $0{ }^{\circ} \mathrm{C}$. After continuous stirring for $6 \mathrm{~h}$ at room temperature, the reaction mixture was diluted with $\mathrm{CH}_{2} \mathrm{Cl}_{2}(30 \mathrm{ml})$, and dried over $\mathrm{MgSO}_{4}$. Evaporation of the solvent left a residue which was chromatographed on silica gel with hexaneEtOAc $(1: 4)$ to give the diol $(3.98 \mathrm{~g}, 90 \%)$ as a colorless oil. ${ }^{1} \mathrm{H}$ NMR $(300 \mathrm{MHz}$, $\left.\mathrm{CDCl}_{3}\right): \delta 4.86(1 \mathrm{H}, \mathrm{d}, J=6.7 \mathrm{~Hz}), 4.79(1 \mathrm{H}, \mathrm{d}, J=6.7 \mathrm{~Hz}), 4.07-4.05(1 \mathrm{H}, \mathrm{m}), 3.88-3.44$ $(8 \mathrm{H}, \mathrm{m}), 3.40(3 \mathrm{H}, \mathrm{s}), 1.20(3 \mathrm{H}, \mathrm{d}, J=6.3 \mathrm{~Hz}), 0.89(9 \mathrm{H}, \mathrm{s}), 0.08(6 \mathrm{H}, \mathrm{s})$; IR (neat): 3446 $\mathrm{cm}^{-1}$; MS (EI) $m / z 338\left(\mathrm{M}^{+}\right)$; HRMS (EI) Calcd for $\mathrm{C}_{15} \mathrm{H}_{34} \mathrm{O}_{6} \mathrm{Si}$ : $338.2125\left(\mathrm{M}^{+}\right)$, found: 338.2155 .

A solution of sodium periodate $(4.28 \mathrm{~g}, 20 \mathrm{mmol})$ and tetra- $n$-butylammonium bromide (358 $\mathrm{mg}, 1.1 \mathrm{mmol})$ in water $(22 \mathrm{ml})$ was added to a solution of the diol $(3.76 \mathrm{~g}, 11$ $\mathrm{mmol})$ in $\mathrm{CH}_{2} \mathrm{Cl}_{2}(23 \mathrm{ml})$ at $0{ }^{\circ} \mathrm{C}$. After continuous stirring for $3 \mathrm{~h}$ at room temperature, the reaction mixture was diluted with $\mathrm{CH}_{2} \mathrm{Cl}_{2}(30 \mathrm{ml})$, washed with water and brine successively, and dried over $\mathrm{MgSO}_{4}$. Evaporation of the solvent afforded a residue which was chromatographed on silica gel with hexane-EtOAc $(2: 1)$ to give the aldehyde $10(2.77 \mathrm{~g}, 81 \%)$ as a colorless oil. ${ }^{1} \mathrm{H}$ NMR $\left(300 \mathrm{MHz}, \mathrm{CDCl}_{3}\right): \delta 9.67(1 \mathrm{H}, \mathrm{d}$ $J=1.8 \mathrm{~Hz}), 4.82(2 \mathrm{H}, \mathrm{s}), 4.19-4.10(1 \mathrm{H}, \mathrm{m}), 3.85(1 \mathrm{H}, \mathrm{dd}, J=4.4,1.8 \mathrm{~Hz}), 3.82-3.70(2 \mathrm{H}$, m), 3.54-3.52 (2H, m), $3.38(3 \mathrm{H}, \mathrm{s}), 1.22(3 \mathrm{H}, \mathrm{d}, J=6.3 \mathrm{~Hz}), 0.87(9 \mathrm{H}, \mathrm{s}), 0.07(3 \mathrm{H}, \mathrm{s})$, $0.06(3 \mathrm{H}, \mathrm{s}) ;{ }^{13} \mathrm{C}$ NMR $\left(75 \mathrm{MHz}, \mathrm{CDCl}_{3}\right): \delta 202.28,95.79,85.66,71.76,69.74,67.69$, 59.19, 25.93, 20.30, 18.23, -4.28, -4.67; IR (neat): $1736 \mathrm{~cm}^{-1}$; MS (EI) $\mathrm{m} / z .306\left(\mathrm{M}^{+}\right)$; HRMS (EI) Calcd for $\mathrm{C}_{14} \mathrm{H}_{30} \mathrm{O}_{5} \mathrm{Si}: 306.1863\left(\mathrm{M}^{+}\right)$, found: 306.1860 ; $[\alpha]_{\mathrm{D}}{ }^{25}+11.4$ $\left(c=1.37, \mathrm{CHCl}_{3}\right)$.

Eth yl (4R,5S)-5-(tert-Butyldimethylsilyloxy)-4-[(2-methoxyethoxy)methoxy]-2hexenoate (11). DBU $(1.49 \mathrm{ml}, 11 \mathrm{mmol})$ was added to a stirred suspension of ethyl diethylphosphonoacetate $(2.16 \mathrm{ml}, 11 \mathrm{mmol})$ and $\mathrm{LiCl}(112 \mathrm{mg}, 2.6 \mathrm{mmol})$ in $\mathrm{MeCN}$ $(90 \mathrm{ml})$ at $0{ }^{\circ} \mathrm{C}$ under Ar atmosphere. After continuous stirring for $30 \mathrm{~min}$ at room 
temperature, a solution of the aldehyde 10 (2.75 g, $8.9 \mathrm{mmol})$ in $\mathrm{MeCN}$ (10 ml) was added dropwise to the solution at $0{ }^{\circ} \mathrm{C}$. After stirring was continued for $1 \mathrm{~h}$ at room temperature, the solvent was evaporated off. The residue was dissolved in ether, and the organic layer was washed successively with water, $10 \% \mathrm{HCl}$, sat. $\mathrm{NaHCO}_{3}$, and brine, and dried over $\mathrm{MgSO}_{4}$. The residue resulting from evaporation of the solvent was chromatographed on silica gel with hexane-EtOAc $(2: 1)$ to give the ethyl ester 11 (3.21 $\mathrm{g}, 95 \%)$ as a colorless oil. ${ }^{1} \mathrm{H} \mathrm{NMR}\left(300 \mathrm{MHz}, \mathrm{CDCl}_{3}\right): \delta 6.84(1 \mathrm{H}$, dd, J=16, $6.3 \mathrm{~Hz})$, $5.99(1 \mathrm{H}, \mathrm{dd}, J=16,1.3 \mathrm{~Hz}), 4.74(2 \mathrm{H}, \mathrm{dd}, J=8.8,6.9 \mathrm{~Hz}), 4.19(2 \mathrm{H}, \mathrm{q}, J=7.1 \mathrm{~Hz}), 4.07-$ $4.04(1 \mathrm{H}, \mathrm{m}), 3.79-3.76(2 \mathrm{H}, \mathrm{m}), 3.65-3.64(1 \mathrm{H}, \mathrm{m}), 3.54-3.52(2 \mathrm{H}, \mathrm{m}), 3.37(3 \mathrm{H}, \mathrm{s})$, $1.28(3 \mathrm{H}, \mathrm{t}, J=7.1 \mathrm{~Hz}), 1.16(3 \mathrm{H}, \mathrm{d}, J=6.0 \mathrm{~Hz}), 0.86(9 \mathrm{H}, \mathrm{s}), 0.04(3 \mathrm{H}, \mathrm{s}), 0.02(3 \mathrm{H}, \mathrm{s})$; ${ }^{13} \mathrm{C}$ NMR (75 MHz, $\left.\mathrm{CDCl}_{3}\right): \delta 166.04,145.69,123.47,94.17,80.11,71.86,70.81$, 67.41, 60.58, 59.23, 26.02, 20.20, 18.31, 14.52, -4.26, -4.49; IR (neat): 1724, $1658 \mathrm{~cm}^{-1}$; MS (EI) $m / z 377\left(\mathrm{M}^{+}+1\right), 361\left(\mathrm{M}^{+}-15\right)$; HRMS (EI) Calcd for $\mathrm{C}_{18} \mathrm{H}_{37} \mathrm{O}_{6} \mathrm{Si}: 377.2364$ $\left(\mathrm{M}^{+}+\mathrm{H}\right)$, found: 377.2364, Calcd for $\mathrm{C}_{17} \mathrm{H}_{33} \mathrm{O}_{6} \mathrm{Si}: 361.2047\left(\mathrm{M}^{+}-\mathrm{CH}_{3}\right)$, found: 361.2047; $[\alpha]_{\mathrm{D}}^{25}-41.9\left(c=1.11, \mathrm{CHCl}_{3}\right)$.

(4R,5S)-5-(tert-Butyldimethylsilyloxy)-4-[(2-methoxyethoxy)methoxy]-2-hexenoic Acid (6). A solution of the ethyl ester $11(3.05 \mathrm{~g}, 8.1 \mathrm{mmol})$ and $0.2 \mathrm{M} \mathrm{NaOH}(16 \mathrm{ml})$ in $\mathrm{MeOH} / \mathrm{THF}(3: 1,65 \mathrm{ml})$ was stirred for $12 \mathrm{~h}$ at room temperature, and then acidified with $10 \% \mathrm{HCl}$ at $0{ }^{\circ} \mathrm{C}$. The mixture was extracted with $\mathrm{CHCl}_{3}$ and combined extracts were dried over $\mathrm{MgSO}_{4}$. Evaporation of the solvent followed by chromatography on silica gel with $\mathrm{CHCl}_{3}-\mathrm{MeOH}(9: 1)$ afforded the carboxylic acid $6(2.79 \mathrm{~g}, 99 \%)$ as a colorless oil. ${ }^{1} \mathrm{H}$ NMR $\left(300 \mathrm{MHz}, \mathrm{CDCl}_{3}\right): \delta 6.98(1 \mathrm{H}, \mathrm{dd}, J=16,6.3 \mathrm{~Hz}), 6.02(1 \mathrm{H}$, dd, $J=11,1.2 \mathrm{~Hz}), 4.75(1 \mathrm{H}, \mathrm{d}, J=6.9 \mathrm{~Hz}), 4.71(1 \mathrm{H}, \mathrm{d}, J=6.9 \mathrm{~Hz}), 4.11-4.08(1 \mathrm{H}, \mathrm{m}), 3.85-$ $3.83(1 \mathrm{H}, \mathrm{m}), 3.78-3.74(1 \mathrm{H}, \mathrm{m}), 3.67-3.64(1 \mathrm{H}, \mathrm{m}), 3.56-3.53(2 \mathrm{H}, \mathrm{m}), 3.38(3 \mathrm{H}, \mathrm{s})$, $1.16(3 \mathrm{H}, \mathrm{d}, J=6.3 \mathrm{~Hz}), 0.86(9 \mathrm{H}, \mathrm{s}), 0.04(3 \mathrm{H}, \mathrm{s}), 0.03(3 \mathrm{H}, \mathrm{s}) ;{ }^{13} \mathrm{C}$ NMR $(75 \mathrm{MHz}$, $\left.\mathrm{CDCl}_{3}\right): \delta 170.99,148.46,122.62,94.33,80.15,71.83,70.74,67.46,59.21,26.01$, 20.22, 18.29, -4.30, -4.49; IR (neat): 2933, 1703, $1657 \mathrm{~cm}^{-1} ;[\alpha]_{\mathrm{D}}{ }^{24}-36.3(c=1.31$, $\left.\mathrm{CHCl}_{3}\right)\left[\right.$ lit. $\left.^{2 \mathrm{a}}[\alpha]_{\mathrm{D}}^{22}-30.0\left(c=0.62, \mathrm{CHCl}_{3}\right)\right]$.

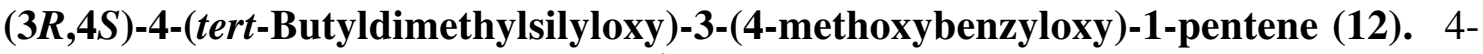
Methoxybenzyl trifluoroacetimidate ${ }^{6)}(5.17 \mathrm{~g}, 22 \mathrm{mmol})$ and pyridinium $p$-toluene sulfonate $(420 \mathrm{mg}, 1.7 \mathrm{mmol})$ were added successively to a stirred solution of the alcohol 7 (2.40 g, $11 \mathrm{mmol})$ in $\mathrm{CH}_{2} \mathrm{Cl}_{2}(35 \mathrm{ml})$ at $0{ }^{\circ} \mathrm{C}$ under Ar atmosphere. After continuous stirring for $24 \mathrm{~h}$ at room temperature, the reaction mixture was diluted with $\mathrm{CH}_{2} \mathrm{Cl}_{2}(25 \mathrm{ml})$, washed with water, $10 \% \mathrm{HCl}$, sat. $\mathrm{NaHCO}_{3}$ and brine successively, and dried over $\mathrm{MgSO}_{4}$. Evaporation of the solvent afforded a residue which was chromatographed on silica gel with hexane-EtOAc $(10: 1)$ to give PMB ether $12(1.74$ g, 47\%, conversion yield 95\%) as a colorless oil. ${ }^{1} \mathrm{H}$ NMR $\left(300 \mathrm{MHz}, \mathrm{CDCl}_{3}\right): \delta 7.22$ $(2 \mathrm{H}, \mathrm{d}, J=8.6 \mathrm{~Hz}), 6.82(2 \mathrm{H}, \mathrm{d}, J=8.6 \mathrm{~Hz}), 5.75(1 \mathrm{H}, \mathrm{ddd}, J=17,10,7.4 \mathrm{~Hz}), 5.25(1 \mathrm{H}$, d, $J=10 \mathrm{~Hz}), 5.20(1 \mathrm{H}, \mathrm{d}, J=17 \mathrm{~Hz}), 4.52(1 \mathrm{H}, \mathrm{d}, J=11 \mathrm{~Hz}), 4.30(1 \mathrm{H}, \mathrm{d}, J=11 \mathrm{~Hz}), 3.76-$ $3.74(1 \mathrm{H}, \mathrm{m}), 3.76(3 \mathrm{H}, \mathrm{s}), 3.51(1 \mathrm{H}, \mathrm{dd}, J=7.5,5.3 \mathrm{~Hz}), 1.13(3 \mathrm{H}, \mathrm{d}, J=6.3 \mathrm{~Hz}), 0.85$ $(9 \mathrm{H}, \mathrm{s}), 0.10(3 \mathrm{H}, \mathrm{s}), 0.08(3 \mathrm{H}, \mathrm{s}) ;{ }^{13} \mathrm{C} \mathrm{NMR}\left(75 \mathrm{MHz}, \mathrm{CDCl}_{3}\right): \delta 159.01,136.64$, $130.92,129.37,118.56,113.73,85.00,71.08,70.32,55.41,26.14,20.52,18.40,-4.18$, 4.25; IR (neat): $1613 \mathrm{~cm}^{-1}$; MS (EI) $\mathrm{m} / z 336\left(\mathrm{M}^{+}\right)$; HRMS (EI) Calcd for $\mathrm{C}_{19} \mathrm{H}_{32} \mathrm{O}_{3} \mathrm{Si}$ : 336.2121 $\left(\mathrm{M}^{+}\right)$, found: $336.2141 ;[\alpha]_{\mathrm{D}}{ }^{26}-22.3\left(c=1.50, \mathrm{CHCl}_{3}\right)$. 
(3R,4S)-4-Hydroxy-3-(4-methoxybenzyloxy)-1-pentene (13). A $1 \mathrm{M}$ solution of tetra$n$-butylammonium fluoride in THF $(0.58 \mathrm{ml}, 0.58 \mathrm{mmol})$ was added to a stirred solution of the PMB ether $12(98.0 \mathrm{mg}, 0.29 \mathrm{mmol})$ in THF $(0.6 \mathrm{ml})$ at room temperature under Ar atmosphere, and the mixture was stirred for $6 \mathrm{~h}$ at room temperature. The reaction mixture was diluted with ether, and the organic layer was washed with water and brine, and dried over $\mathrm{MgSO}_{4}$. Evaporation of the solvent left a residue which was chromatographed on silica gel with hexane-EtOAc $(2: 1)$ to give the alcohol $13(62 \mathrm{mg}$, $96 \%)$ as a colorless oil. ${ }^{1} \mathrm{H}$ NMR $\left(300 \mathrm{MHz}, \mathrm{CDCl}_{3}\right): \delta 7.23(2 \mathrm{H}, \mathrm{d}, J=8.6 \mathrm{~Hz}), 6.87$ $(2 \mathrm{H}, \mathrm{d}, J=8.6 \mathrm{~Hz}), 5.82(1 \mathrm{H}, \mathrm{ddd}, J=17,10,8.0 \mathrm{~Hz}), 5.38(1 \mathrm{H}, \mathrm{dd}, J=10,1.9 \mathrm{~Hz}), 5.29$ $(1 \mathrm{H}, \mathrm{ddd}, J=17,1.9,0.8 \mathrm{~Hz}), 4.56(1 \mathrm{H}, \mathrm{d}, J=11 \mathrm{~Hz}), 4.30(1 \mathrm{H}, \mathrm{d}, J=11 \mathrm{~Hz}), 3.86-3.84$ $(1 \mathrm{H}, \mathrm{m}), 3.79(3 \mathrm{H}, \mathrm{s}), 3.64(1 \mathrm{H}, \mathrm{dd}, J=8.0,3.8 \mathrm{~Hz}), 1.12(3 \mathrm{H}, \mathrm{d}, J=6.6 \mathrm{~Hz}) ;{ }^{13} \mathrm{C} \mathrm{NMR}$ $\left(75 \mathrm{MHz}, \mathrm{CDCl}_{3}\right): \delta 159.13,134.69,130.35,129.41,120.17,113.83,84.09,70.05$, 69.37, 55.39, 18.21; IR (neat): 3449, $1612 \mathrm{~cm}^{-1}$; MS (EI) $\mathrm{m} / z 222\left(\mathrm{M}^{+}\right)$; HRMS (EI) Calcd for $\mathrm{C}_{13} \mathrm{H}_{18} \mathrm{O}_{3}: 222.1256\left(\mathrm{M}^{+}\right)$, found : $222.1221 ;[\alpha]_{\mathrm{D}}{ }^{24}-45.2\left(c=1.28, \mathrm{CHCl}_{3}\right)$.

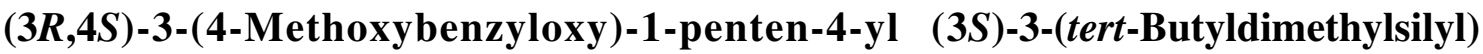
butanoate (14). 1-Ethyl-3-(3-dimethylaminopropyl)carbodiimide hydrochloride (2.28 g, $12 \mathrm{mmol})$ was added to a stirred solution of the alcohol 13 (1.33 g, $6.0 \mathrm{mmol}),(S)-3-$ (tert-butyldimethylsilyloxy)butanoic acid ${ }^{4}$ (2.56 g, $\left.12 \mathrm{mmol}\right)$, and 4-dimethylamino pyridine $(73.3 \mathrm{mg}, 0.60 \mathrm{mmol})$ in $\mathrm{CH}_{2} \mathrm{Cl}_{2}(50 \mathrm{ml})$ at $0{ }^{\circ} \mathrm{C}$ under $\mathrm{Ar}$ atmosphere. After continuous stirring for $12 \mathrm{~h}$ at room temperature, the solvent was evaporated to leave a residue which was triturated with ether and filtered through celite. Evaporation of the solvent followed by chromatography on silica gel with hexane-EtOAc $(4: 1)$ afforded the ester $14(2.49 \mathrm{~g}, 99 \%)$ as a colorless oil. ${ }^{1} \mathrm{H} \mathrm{NMR}\left(300 \mathrm{MHz}, \mathrm{CDCl}_{3}\right): \delta 7.25(2 \mathrm{H}$, d, $J=8.8 \mathrm{~Hz}), 6.87(2 \mathrm{H}, \mathrm{d}, J=8.8 \mathrm{~Hz}), 5.74(1 \mathrm{H}, \mathrm{ddd}, J=18,11,7.4 \mathrm{~Hz}), 5.32(1 \mathrm{H}, \mathrm{d}$, $J=11 \mathrm{~Hz}), 5.28(1 \mathrm{H}, \mathrm{d}, J=18 \mathrm{~Hz}), 4.97-4.95(1 \mathrm{H}, \mathrm{m}) 4.55(1 \mathrm{H}, \mathrm{d}, J=12 \mathrm{~Hz}), 4.35(1 \mathrm{H}, \mathrm{d}$, $J=12 \mathrm{~Hz}), 4.25-4.23(1 \mathrm{H}, \mathrm{m}), 3.80(3 \mathrm{H}, \mathrm{s}), 3.73-3.71(1 \mathrm{H}, \mathrm{m}), 2.47(1 \mathrm{H}, \mathrm{dd}, J=15,6.3$ $\mathrm{Hz}), 2.34(1 \mathrm{H}, \mathrm{dd}, J=15,6.3 \mathrm{~Hz}), 1.22(3 \mathrm{H}, \mathrm{d}, J=6.6 \mathrm{~Hz}), 1.18(3 \mathrm{H}, \mathrm{d}, J=6.0 \mathrm{~Hz}), 0.86$ $(9 \mathrm{H}, \mathrm{s}), 0.06(3 \mathrm{H}, \mathrm{s}), 0.05(3 \mathrm{H}, \mathrm{s}) ;{ }^{13} \mathrm{C} \mathrm{NMR}\left(75 \mathrm{MHz}, \mathrm{CDCl}_{3}\right): \delta 170.34,159.10$, 135.07, 130.43, 129.28, 119.43, 113.78, 81.71, 71.99, 70.32, 65.84, 55.41, 45.29, 26.04, 25.96, 24.02, 18.26, 15.65, -4.25, -4.59; IR (neat): 1737, $1613 \mathrm{~cm}^{-1}$; MS (EI) $\mathrm{m} / z 422$ $\left(\mathrm{M}^{+}\right)$; HRMS (EI) Calcd for $\mathrm{C}_{23} \mathrm{H}_{38} \mathrm{O}_{5} \mathrm{Si}: 422.2489\left(\mathrm{M}^{+}\right)$, found: 422.2515 ; $[\alpha]_{\mathrm{D}}{ }^{27}-19.7$ $\left(c=0.98, \mathrm{CHCl}_{3}\right)$.

(3R,4S)-3-(4-Methoxybenzyloxy)-1-penten-4-yl (3S)-3-hydroxybutanoate (5). A $1 \mathrm{M}$ solution of tetra- $n$-butylammonium fluoride in THF $(12.0 \mathrm{ml}, 12 \mathrm{mmol})$ was added to a stirred solution of the silyl ether $14(2.51 \mathrm{~g}, 6.0 \mathrm{mmol})$ in THF $(12 \mathrm{ml})$ at room temperature under Ar atmosphere, and the mixture was stirred for $5 \mathrm{~h}$ at room temperature. The solvent was evaporated off to leave a residue, which was dissolved in ether and the resulting organic layer was washed with water and brine, and dried over $\mathrm{MgSO}_{4}$. Evaporation of the solvent left a residue which was chromatographed on silica gel with hexane-EtOAc $(1: 1)$ to give the alcohol $5(1.75 \mathrm{~g}, 95 \%)$ as a colorless oil. ${ }^{1} \mathrm{H}$ NMR $\left(300 \mathrm{MHz}, \mathrm{CDCl}_{3}\right): \delta 7.24(2 \mathrm{H}, \mathrm{d}, J=8.8 \mathrm{~Hz}), 6.86(2 \mathrm{H}, \mathrm{d}, J=8.8 \mathrm{~Hz}), 5.75(1 \mathrm{H}$, ddd, $J=17,10,7.7 \mathrm{~Hz}), 5.34(1 \mathrm{H}, \mathrm{d}, J=10 \mathrm{~Hz}), 5.29(1 \mathrm{H}, \mathrm{d}, J=17 \mathrm{~Hz}), 5.05(1 \mathrm{H}, \mathrm{dq}$, $J=6.4,4.4 \mathrm{~Hz}), 4.56(1 \mathrm{H}, \mathrm{d}, J=12 \mathrm{~Hz}), 4.33(1 \mathrm{H}, \mathrm{d}, J=12 \mathrm{~Hz}), 4.13-4.11(1 \mathrm{H}, \mathrm{m}), 3.79$ $(3 \mathrm{H}, \mathrm{s}), 3.76-3.74(1 \mathrm{H}, \mathrm{m}), 3.17(1 \mathrm{H}, \mathrm{br}), 2.48-2.33(2 \mathrm{H}, \mathrm{m}), 1.22(3 \mathrm{H}, \mathrm{d}, J=6.4 \mathrm{~Hz})$, $1.20(3 \mathrm{H}, \mathrm{d}, J=6.4 \mathrm{~Hz}) ;{ }^{13} \mathrm{C} \mathrm{NMR}\left(75 \mathrm{MHz} \mathrm{CDCl}_{3}\right): \delta 172.01,159.09,134.54,130.06$, 
$129.38,129.33,119.87,113.75,81.39,72.04,70.15,64.47,55.34,43.53,22.66,15.70$; IR (neat): 3449, 1730, $1613 \mathrm{~cm}^{-1}$; MS (EI) $\mathrm{m} / 2308\left(\mathrm{M}^{+}\right)$; HRMS (EI) Calcd for $\mathrm{C}_{17} \mathrm{H}_{24} \mathrm{O}_{5}: 308.1624\left(\mathrm{M}^{+}\right)$, found: $308.1652 ;[\alpha]_{\mathrm{D}}{ }^{24}-32.3\left(c=1.04, \mathrm{CHCl}_{3}\right)$.

(-)-1-[3-(4-Methoxybenzyloxy)-1-penten-4-yloxycarbonyl]prop-2-yl 5-(tertButyldimethylsilyloxy)-4-[(2-methoxyethoxy)methoxy]-2-hexenoate (15). To a solution of the carboxylic acid $6(5.1 \mathrm{~g}, 14.6 \mathrm{mmol})$ and $\mathrm{Et}_{3} \mathrm{~N}(4.06 \mathrm{ml}, 29.2 \mathrm{mmol})$ in toluene $(150 \mathrm{ml})$ was added 2,4,6-trichlorobenzoyl chloride $(3.03 \mathrm{ml}, 19.4 \mathrm{mmol})$ at room temperature under Ar atmosphere, and the resulting mixture was stirred at room temperature for $1 \mathrm{~h}$. The alcohol 5 (3.00 g, $9.72 \mathrm{mmol})$ and DMAP (1.43 g, $11.7 \mathrm{mmol})$ were added, and the reaction mixture was stirred for $1 \mathrm{~h}$. After the reaction completed, the mixture was washed with sat. $\mathrm{NaHCO}_{3}$ and brine, and dried over $\mathrm{MgSO}_{4}$. The solvent was evaporated off to leave a residue which was chromatographed on silica gel with hexane-EtOAc $(2: 1)$ to afford the ester $15(6.14 \mathrm{~g}, 99 \%)$ as a colorless oil. ${ }^{1} \mathrm{H}$ NMR $\left(300 \mathrm{MHz}, \mathrm{CDCl}_{3}\right): \delta 7.24(2 \mathrm{H}, \mathrm{d}, J=8.8 \mathrm{~Hz}), 6.87(2 \mathrm{H}, \mathrm{d}, J=8.8 \mathrm{~Hz}), 6.82(1 \mathrm{H}, \mathrm{d}$, $J=16 \mathrm{~Hz}), 5.95(1 \mathrm{H}, \mathrm{dd}, J=16,1.2 \mathrm{~Hz}), 5.73(1 \mathrm{H}, \mathrm{ddd}, J=17,10,7.5 \mathrm{~Hz}), 5.32(1 \mathrm{H}, \mathrm{d}$, $J=10 \mathrm{~Hz}), 5.32-5.29(1 \mathrm{H}, \mathrm{m}), 5.28(1 \mathrm{H}, \mathrm{d}, J=17 \mathrm{~Hz}), 4.99-4.97(1 \mathrm{H}, \mathrm{m}), 4.71(2 \mathrm{H}, \mathrm{dd}$, $J=9.9,6.9 \mathrm{~Hz}), 4.54(1 \mathrm{H}, \mathrm{d}, J=12 \mathrm{~Hz}), 4.33(1 \mathrm{H}, \mathrm{d}, J=12 \mathrm{~Hz}), 4.06-4.04(1 \mathrm{H}, \mathrm{m}), 3.85-$ $3.71(2 \mathrm{H}, \mathrm{m}), 3.80(3 \mathrm{H}, \mathrm{s}), 3.67-3.60(1 \mathrm{H}, \mathrm{m}), 3.54-3.51(2 \mathrm{H}, \mathrm{m}), 3.38(3 \mathrm{H}, \mathrm{s}), 2.66$ $(1 \mathrm{H}, \mathrm{dd}, J=15,6.9 \mathrm{~Hz}), 2.48(1 \mathrm{H}, \mathrm{dd}, J=16,6.9 \mathrm{~Hz}), 1.29(3 \mathrm{H}, \mathrm{d}, J=6.3 \mathrm{~Hz}), 1.18(3 \mathrm{H}$, d, $J=6.6 \mathrm{~Hz}), 1.15(3 \mathrm{H}, \mathrm{d}, J=6.3 \mathrm{~Hz}), 0.87(9 \mathrm{H}, \mathrm{s}), 0.06(3 \mathrm{H}, \mathrm{s}), 0.04(3 \mathrm{H}, \mathrm{s}) ;{ }^{13} \mathrm{C}$ NMR $\left(75 \mathrm{MHz} \mathrm{CDCl}_{3}\right): \delta 169.26,164.96,159.07,145.85,134.82,130.24,129.28,123.43$, $119.72,113.75,94.01,81.55,79.95,72.15,71.76,70.68,70.16,67.49,67.31,59.14$, 55.36, 41.31, 25.98, 20.10, 19.99, 18.23, 15.77, -4.35, -4.54; IR (neat): 1730, 1657, $1613 \mathrm{~cm}^{-1}$; MS (EI) $\mathrm{m} / z 638\left(\mathrm{M}^{+}\right)$; HRMS (EI) Calcd for $\mathrm{C}_{33} \mathrm{H}_{54} \mathrm{O}_{10} \mathrm{Si}: 638.3486\left(\mathrm{M}^{+}\right)$, found : $638.3467 ;[\alpha]_{\mathrm{D}}{ }^{26}-39.7\left(c=0.95, \mathrm{CHCl}_{3}\right)$.

(-)-1-[3-(4-Methoxybenzyloxy)-1-penten-4-yloxycarbonyl]prop-2-yl 5-Hydroxy-4[(2-methoxyethoxy)methoxy]-2-hexenoate (16). A 1 M solution of tetra- $n$ butylammonium fluoride in THF $(19.6 \mathrm{ml}, 20 \mathrm{mmol})$ was added to a stirred solution of the silyl ether 15 (626 mg, $0.98 \mathrm{mmol})$ and acetic acid $(1.23 \mathrm{ml}, 22 \mathrm{mmol})$ in THF (45 $\mathrm{ml}$ ) at room temperature under Ar atmosphere, and the mixture was stirred for 5 days at room temperature. The ethereal solution of the residue resulting from the evaporation of the solvent was washed with water, sat. $\mathrm{NaHCO}_{3}$, and brine, and dried over $\mathrm{MgSO}_{4}$. Evaporation of the solvent left a residue which was chromatographed on silica gel with hexane-EtOAc $(1: 2)$ to give the alcohol $16(433 \mathrm{mg}, 85 \%)$ as a colorless oil. ${ }^{1} \mathrm{H}$ NMR $\left(300 \mathrm{MHz}, \mathrm{CDCl}_{3}\right): \delta 7.24(2 \mathrm{H}, \mathrm{d}, J=8.2 \mathrm{~Hz}), 6.90-6.80(3 \mathrm{H}, \mathrm{m}), 6.01(1 \mathrm{H}, \mathrm{d}, J=16 \mathrm{~Hz})$, $5.74(1 \mathrm{H}$, ddd, $J=17,10,7.4 \mathrm{~Hz}), 5.30(1 \mathrm{H}, \mathrm{d}, J=10 \mathrm{~Hz}), 5.30-5.28(1 \mathrm{H}, \mathrm{m}), 5.28(1 \mathrm{H}, \mathrm{d}$, $J=17 \mathrm{~Hz}), 4.99-4.96(1 \mathrm{H}, \mathrm{m}), 4.76(1 \mathrm{H}, \mathrm{d}, J=7.4 \mathrm{~Hz}), 4.69(1 \mathrm{H}, \mathrm{d}, J=7.4 \mathrm{~Hz}), 4.54(1 \mathrm{H}$, d, $J=11 \mathrm{~Hz}), 4.32(1 \mathrm{H}, \mathrm{d}, J=11 \mathrm{~Hz}), 4.22-4.20(1 \mathrm{H}, \mathrm{m}), 3.95-3.62(4 \mathrm{H}, \mathrm{m}), 3.79(3 \mathrm{H}, \mathrm{s})$, 3.55-3.48 (2H, m), $3.37(3 \mathrm{H}, \mathrm{s}), 3.16(1 \mathrm{H}, \mathrm{br}), 2.65(1 \mathrm{H}, \mathrm{dd}, J=15,7.1 \mathrm{~Hz}), 2.51(1 \mathrm{H}$, $\mathrm{dd}, J=15,6.0 \mathrm{~Hz}), 1.31(3 \mathrm{H}, \mathrm{d}, J=5.8 \mathrm{~Hz}), 1.18(3 \mathrm{H}, \mathrm{d}, J=6.3 \mathrm{~Hz}), 1.13(3 \mathrm{H}, \mathrm{d}, J=6.0$ $\mathrm{Hz}) ;{ }^{13} \mathrm{C}$ NMR $\left(75 \mathrm{MHz}, \mathrm{CDCl}_{3}\right): \delta 169.13,164.70,158.90,144.05,134.61,130.06$, $129.10,123.55,119.54,113.57,94.24,81.34,80.60$, , 71.96, 71.59, 69.97, 68.87, 67.40, 58.87, 55.15, 41.09, 19.83, 17.79, 15.52, 14.23; IR (neat): 3465, 1733, 1657, $1613 \mathrm{~cm}^{-1}$; MS (EI) $m / z 524\left(\mathrm{M}^{+}\right)$; HRMS (EI) Calcd for $\mathrm{C}_{27} \mathrm{H}_{40} \mathrm{O}_{10}: 524.2622\left(\mathrm{M}^{+}\right)$, found: 524.2674; $[\alpha]_{\mathrm{D}}{ }^{25}-53.1\left(c=0.87, \mathrm{CHCl}_{3}\right)$. 
(-)-1-[3-(4-Methoxybenzyloxy)-1-penten-4-yloxycarbonyl]prop-2-yl 5-Acryloyloxy4-[(2-methoxyethoxy)methoxy]-2-hexenoate (4). Acryloyl chloride $(0.263 \mathrm{ml}, 3.2$ mmol) was added dropwise to a stirred solution of the alcohol 16 (425 $\mathrm{mg}, 0.81 \mathrm{mmol})$ and $\mathrm{N}, \mathrm{N}$-diisopropylethylamine $(0.705 \mathrm{ml}, 4.1 \mathrm{mmol})$ in $\mathrm{CH}_{2} \mathrm{Cl}_{2}(8 \mathrm{ml})$ at $0{ }^{\circ} \mathrm{C}$ under $\mathrm{Ar}$ atmosphere. After continuous stirring for $1 \mathrm{~h}$ at room temperature, the reaction mixture was diluted with $\mathrm{CH}_{2} \mathrm{Cl}_{2}(10 \mathrm{ml})$, washed with water, $10 \% \mathrm{HCl}$, sat. $\mathrm{NaHCO}_{3}$, and brine successively, and dried over $\mathrm{MgSO}_{4}$. Evaporation of the solvent afforded a residue which was chromatographed on silica gel with hexane-EtOAc $(2: 1)$ to give the ester 4 (444 mg, 95\%) as a colorless oil. ${ }^{1} \mathrm{H}$ NMR $\left(300 \mathrm{MHz}, \mathrm{CDCl}_{3}\right): \delta 7.24(2 \mathrm{H}, \mathrm{d}, J=8.6 \mathrm{~Hz})$, $6.87(2 \mathrm{H}, \mathrm{d}, J=8.6 \mathrm{~Hz}), 6.80(1 \mathrm{H}, \mathrm{d}, J=16 \mathrm{~Hz}), 6.39(1 \mathrm{H}, \mathrm{d}, J=17 \mathrm{~Hz}), 6.13(1 \mathrm{H}, \mathrm{d}, J=10$ $\mathrm{Hz}), 6.03(1 \mathrm{H}, \mathrm{d}, J=16 \mathrm{~Hz}), 5.83(1 \mathrm{H}, \mathrm{d}, J=10 \mathrm{~Hz}), 5.74(1 \mathrm{H}$, ddd, $J=17,10,7.1 \mathrm{~Hz})$, $5.34(1 \mathrm{H}, \mathrm{d}, J=10 \mathrm{~Hz}), 5.34-5.32(1 \mathrm{H}, \mathrm{m}), 5.28(1 \mathrm{H}, \mathrm{d}, J=17 \mathrm{~Hz}), 5.08-5.05(1 \mathrm{H}, \mathrm{m})$, 4.98-4.96 $(1 \mathrm{H}, \mathrm{m}), 4.74(1 \mathrm{H}, \mathrm{d}, J=6.9 \mathrm{~Hz}), 4.69(1 \mathrm{H}, \mathrm{d}, J=6.9 \mathrm{~Hz}), 4.54(1 \mathrm{H}, \mathrm{d}, J=12$ $\mathrm{Hz}), 4.40-4.38(1 \mathrm{H}, \mathrm{m}), 4.33(1 \mathrm{H}, \mathrm{d}, J=12 \mathrm{~Hz}), 3.84-3.72(2 \mathrm{H}, \mathrm{m}), 3.79(3 \mathrm{H}, \mathrm{s}), 3.66-$ $3.60(1 \mathrm{H}, \mathrm{m}), 3.54-3.50(2 \mathrm{H}, \mathrm{m}), 3.36(3 \mathrm{H}, \mathrm{s}), 2.66(1 \mathrm{H}, \mathrm{dd}, J=15,7.3 \mathrm{~Hz}), 2.51(1 \mathrm{H}$, dd, $J=15,6.0 \mathrm{~Hz}), 1.31(3 \mathrm{H}, \mathrm{d}, J=6.3 \mathrm{~Hz}), 1.23(3 \mathrm{H}, \mathrm{d}, J=6.6 \mathrm{~Hz}), 1.18(3 \mathrm{H}, \mathrm{d}, J=6.3$ $\mathrm{Hz}) ;{ }^{13} \mathrm{C}$ NMR $\left(75 \mathrm{MHz}, \mathrm{CDCl}_{3}\right): \delta 169.12,165.12,164.63,158.97,143.29,134.67$, $130.95,130.11,129.17,128.31,124.14,119.62,113.64,93.74,81.41,76.52,72.04$, 71.59, 71.41, 70.03, 67.67, 59.00, 55.23, 41.15, 19.89, 15.64, 15.04; IR (neat): 1728, $1657,1614 \mathrm{~cm}^{-1}$; MS (EI) $m / z 547\left(\mathrm{M}^{+}-31\right) ;[\alpha]_{\mathrm{D}}^{25}-50.2\left(c=0.99, \mathrm{CHCl}_{3}\right)$.

Synthesis of 3 by Ring-Closing Metathesis of 4. Grubbs' catalyst (cat. 2) (20.4 mg, $0.024 \mathrm{mmol})$ was added to a solution of $4(14.0 \mathrm{mg}, 0.024 \mathrm{mmol})$ in 1,2-dichloroethane $(24 \mathrm{ml})$ at room temperature under Ar atmosphere. After continuous stirring for 5 days at $80{ }^{\circ} \mathrm{C}$, the solvent was evaporated to afford a residue which was chromatographed on silica gel with hexane-EtOAc $(1: 1)$ to give $3(8.5 \mathrm{mg}, 65 \%)$ as a colorless oil. ${ }^{1} \mathrm{H}$ NMR $\left(300 \mathrm{MHz}, \mathrm{CDCl}_{3}\right): \delta 7.21(2 \mathrm{H}, \mathrm{d}, J=8.5 \mathrm{~Hz}), 6.87(2 \mathrm{H}, \mathrm{d}, J=8.5 \mathrm{~Hz}), 6.75(1 \mathrm{H}$, dd, $J=15,7.4 \mathrm{~Hz}), 6.74(1 \mathrm{H}, \mathrm{dd}, J=15,7.9 \mathrm{~Hz}), 5.99(1 \mathrm{H}, \mathrm{d}, J=15 \mathrm{~Hz}), 5.91(1 \mathrm{H}, \mathrm{d}, J=15$ $\mathrm{Hz}), 5.31-5.29(1 \mathrm{H}, \mathrm{m}), 5.02-4.97(2 \mathrm{H}, \mathrm{m}), 4.74(1 \mathrm{H}, \mathrm{d}, J=7.1 \mathrm{~Hz}), 4.71(1 \mathrm{H}, \mathrm{d}, J=7.1$ $\mathrm{Hz}), 4.53(1 \mathrm{H}, \mathrm{d}, J=11 \mathrm{~Hz}), 4.27(1 \mathrm{H}, \mathrm{d}, J=11 \mathrm{~Hz}), 4.13-4.11(1 \mathrm{H}, \mathrm{m}), 3.80(3 \mathrm{H}, \mathrm{s})$, 3.78-3.63 (3H, m), 3.57-3.54 (2H, m), $3.39(3 \mathrm{H}, \mathrm{s}), 2.56-2.50(2 \mathrm{H}, \mathrm{m}), 1.43(3 \mathrm{H}, \mathrm{d}$, $J=6.3 \mathrm{~Hz}), 1.29(3 \mathrm{H}, \mathrm{d}, J=6.0 \mathrm{~Hz}), 1.27(3 \mathrm{H}, \mathrm{d}, J=6.0 \mathrm{~Hz}) ;{ }^{13} \mathrm{C} \mathrm{NMR}\left(75 \mathrm{MHz}, \mathrm{CDCl}_{3}\right)$ : $\delta 169.46,164.31,164.17,159.33,145.01,129.62,129.25,124.30,124.12,113.86$, 94.11, 79.13, 78.71, 71.91, 71.65, 71.04, 70.81, 67.75, 67.51, 59.14, 55.37, 53.61, 41.17, 19.91, 18.10, 17.98; IR (neat): 1726, $1658 \mathrm{~cm}^{-1}$; MS (EI) $\mathrm{m} / z 550\left(\mathrm{M}^{+}\right)$; HRMS (EI) Calcd for $\mathrm{C}_{28} \mathrm{H}_{38} \mathrm{O}_{11}: 550.2414\left(\mathrm{M}^{+}\right)$, found: 550.2368; $[\alpha]_{\mathrm{D}}{ }^{24}-76.1(c=2.34$, $\left.\mathrm{CHCl}_{3}\right)$.

1-(3-Hydroxy-1-penten-4-yloxycarbonyl)pro p - 2 - y l 5 - A c r y l o y l o x y - 4 - [ ( 2 methoxyethoxy)methoxy]-2-hexenoate (17). A mixture of PMB ether 4 (480 $\mathrm{mg}, 0.83$ mmol) and DDQ (282 mg, $1.25 \mathrm{mmol})$ in $\mathrm{CH}_{2} \mathrm{Cl}_{2} / \mathrm{H}_{2} \mathrm{O}(18: 1,10 \mathrm{ml})$ was stirred at room temperature for $1 \mathrm{~h}$. The precipitate formed was removed by filtration, and the filtrate was concentrated to furnish a gummy mass, which was chromatographed on silica gel with hexane-EtOAc $(1: 1)$ to give the alcohol $17(368 \mathrm{mg}, 97 \%)$ as a colorless oil. ${ }^{1} \mathrm{H}$ NMR $\left(300 \mathrm{MHz}, \mathrm{CDCl}_{3}\right): \delta 6.85(1 \mathrm{H}, \mathrm{dd}, J=16,6.2 \mathrm{~Hz}), 6.41(1 \mathrm{H}, \mathrm{dd}, J=16,1.4$ $\mathrm{Hz}), 6.10(1 \mathrm{H}, \mathrm{dd}, J=17,10 \mathrm{~Hz}), 6.06(1 \mathrm{H}, \mathrm{dd}, J=16,1.4 \mathrm{~Hz}), 5.84(1 \mathrm{H}, \mathrm{dd}, J=10,1.4$ 
Hz), $5.81(1 \mathrm{H}, \mathrm{ddd}, J=17,10,5.8 \mathrm{~Hz}), 5.37-5.35(1 \mathrm{H}, \mathrm{m}), 5.35(1 \mathrm{H}, \mathrm{d}, J=17 \mathrm{~Hz}), 5.25$ $(1 \mathrm{H}, \mathrm{d}, J=17 \mathrm{~Hz}), 5.13-5.10(1 \mathrm{H}, \mathrm{m}), 4.98-4.95(1 \mathrm{H}, \mathrm{m}), 4.76(1 \mathrm{H}, \mathrm{d}, J=7.0 \mathrm{~Hz}), 4.60$ $(1 \mathrm{H}, \mathrm{d}, J=7.0 \mathrm{~Hz}), 4.41-4.39(1 \mathrm{H}, \mathrm{m}), 4.24-4.22(1 \mathrm{H}, \mathrm{m}), 3.79-3.76(1 \mathrm{H}, \mathrm{m}), 3.65-3.63$ $(1 \mathrm{H}, \mathrm{m}), 3.55-3.50(2 \mathrm{H}, \mathrm{m}), 3.38(3 \mathrm{H}, \mathrm{s}), 2.70(1 \mathrm{H}, \mathrm{dd}, J=16,8.0 \mathrm{~Hz}), 2.57(1 \mathrm{H}, \mathrm{dd}$, $J=16,5.2 \mathrm{~Hz}), 1.34(3 \mathrm{H}, \mathrm{d}, J=6.3 \mathrm{~Hz}), 1.25(3 \mathrm{H}, \mathrm{d}, J=6.5 \mathrm{~Hz}), 1.20(3 \mathrm{H}, \mathrm{d}, J=6.3 \mathrm{~Hz})$; ${ }^{13} \mathrm{C} \mathrm{NMR}\left(75 \mathrm{MHz}, \mathrm{CDCl}_{3}\right): \delta 169.73,165.33,164.99,143.71,135.80,131.14,128.41$, $124.10,117.37,93.90,76.67,74.56,73.85,71.70,71.54,67.82,67.35,59.14,41.41$, $20.23,15.14,14.41$.

Synthesis of 18. (Method A) Grubbs' catalyst (cat. 2) (52 mg, $0.06 \mathrm{mmol}$ ) was added to a solution of $17(285 \mathrm{mg}, 0.62 \mathrm{mmol})$ in $\mathrm{CH}_{2} \mathrm{Cl}_{2}(622 \mathrm{ml})$ under Ar atmosphere. After continuous stirring for $24 \mathrm{~h}$ at room temperature, the solvent was evaporated to afford a residue which was chromatographed on silica gel with hexane-AcOEt $(2: 1)$ to give the macrocyclic alcohol $18(207 \mathrm{mg}, 77 \%)$ as a colorless solid. (Method B) A mixture of PMB ether 3 ( $8.0 \mathrm{mg}, 0.015 \mathrm{mmol}$ ) and DDQ (4.95 mg, $0.022 \mathrm{mmol}$ ) in $\mathrm{CH}_{2} \mathrm{Cl}_{2} / \mathrm{H}_{2} \mathrm{O}$ $(18: 1,0.16 \mathrm{ml})$ was stirred at room temperature for $30 \mathrm{~min}$. The precipitate formed was removed by filtration, and the filtrate was concentrated to furnish a gummy mass, which was chromatographed on silica gel with hexane-EtOAc $(2: 1)$ to give $\mathbf{1 8}(6.2 \mathrm{mg}, 99 \%)$ as a colorless solid. mp 94-96 ${ }^{\circ} \mathrm{C} ;{ }^{1} \mathrm{H}$ NMR $\left(300 \mathrm{MHz}, \mathrm{CDCl}_{3}\right): \delta 6.80(1 \mathrm{H}, \mathrm{dd}, J=16$, $5.1 \mathrm{~Hz}), 6.72(1 \mathrm{H}, \mathrm{dd}, J=16,7.1 \mathrm{~Hz}), 5.99(1 \mathrm{H}, \mathrm{dd}, J=16,1.4 \mathrm{~Hz}), 5.93(1 \mathrm{H}, \mathrm{d}, J=16$ $\mathrm{Hz}), 5.28-5.26(1 \mathrm{H}, \mathrm{m}), 4.93-4.83(2 \mathrm{H}, \mathrm{m}), 4.70-4.67(2 \mathrm{H}, \mathrm{m}), 4.15-4.05(2 \mathrm{H}, \mathrm{m}), 3.78-$ $3.71(1 \mathrm{H}, \mathrm{m}), 3.65-3.59(1 \mathrm{H}, \mathrm{m}), 3.53-3.36(2 \mathrm{H}, \mathrm{m}), 3.35(3 \mathrm{H}, \mathrm{s}), 2.97(1 \mathrm{H}, \mathrm{br}), 2.69$ $(1 \mathrm{H}, \mathrm{dd}, J=15,2.9 \mathrm{~Hz}), 2.48(1 \mathrm{H}, \mathrm{dd}, J=15,8.3 \mathrm{~Hz}), 1.37(3 \mathrm{H}, \mathrm{d}, J=6.0 \mathrm{~Hz}), 1.31(3 \mathrm{H}$, d, $J=6.8 \mathrm{~Hz}), 1.29(3 \mathrm{H}, \mathrm{d}, J=6.8 \mathrm{~Hz}) ;{ }^{13} \mathrm{C} \mathrm{NMR}\left(75 \mathrm{MHz}, \mathrm{CDCl}_{3}\right): \delta 169.94,164.61$, $164.38,145.77,144.75,124.54,122.57,94.01,78.66,73.75,72.95,71.67,71.04,67.85$, 67.49, 59.16, 41.01, 19.73, 18.08, 17.72; IR (KBr): 3435, 1711, $1647 \mathrm{~cm}^{-1}$; MS (EI) $\mathrm{m} / \mathrm{z}$ $430\left(\mathrm{M}^{+}\right)$; HRMS (EI) Calcd for $\mathrm{C}_{20} \mathrm{H}_{30} \mathrm{O}_{10}: 430.1839\left(\mathrm{M}^{+}\right)$, found: $430.1882 ;[\alpha]_{\mathrm{D}}{ }^{24}$ $-31.0\left(c=0.88, \mathrm{CHCl}_{3}\right)$.

Macrosphelide A (1). Trifluoroacetic acid $(0.6 \mathrm{ml})$ was added to a solution of MEM ether $18(21.5 \mathrm{mg}, 0.05 \mathrm{mmol})$ in $\mathrm{CH}_{2} \mathrm{Cl}_{2}(0.6 \mathrm{ml})$ at $0{ }^{\circ} \mathrm{C}$ under Ar atmosphere. After continuous stirring for $24 \mathrm{~h}$ at room temperature, the solvent was evaporated to afford a residue which was chromatographed on silica gel with $\mathrm{CH}_{2} \mathrm{Cl}_{2}-\mathrm{EtOAc}(1: 1)$ to give macrosphelide $\mathrm{A}(\mathbf{1})(15.4 \mathrm{mg}, 90 \%)$ as a colorless solid. mp 144-146 ${ }^{\circ} \mathrm{C}$ (colorless needles from $\mathrm{CH}_{2} \mathrm{Cl}_{2}$-hexane, lit. $\left.{ }^{2 \mathrm{a}} \mathrm{mp} 146-147{ }^{\circ} \mathrm{C}\right) ;{ }^{1} \mathrm{H} \mathrm{NMR}\left(500 \mathrm{MHz}, \mathrm{CDCl}_{3}\right): \delta 6.88$ (1H, dd, $J=16,4.9, \mathrm{~Hz}), 6.87(1 \mathrm{H}, \mathrm{dd}, J=15,4.5, \mathrm{~Hz}), 6.05(1 \mathrm{H}, \mathrm{dd}, J=16,1.7, \mathrm{~Hz}), 6.03$ $(1 \mathrm{H}, \mathrm{dd}, J=16,1.7, \mathrm{~Hz}), 5.40-5.37(1 \mathrm{H}, \mathrm{m}), 4.97-4.94(1 \mathrm{H}, \mathrm{m}), 4.88-4.85(1 \mathrm{H}, \mathrm{m}), 4.23$ (1H, brs), $4.15(1 \mathrm{H}, \mathrm{brs}), 2.62(1 \mathrm{H}, \mathrm{dd}, J=15,9.0, \mathrm{~Hz}), 2.57(1 \mathrm{H}, \mathrm{dd}, J=15,3.4, \mathrm{~Hz})$, $1.46(3 \mathrm{H}, \mathrm{d}, J=6.4 \mathrm{~Hz}), 1.37(3 \mathrm{H}, \mathrm{d}, J=6.0 \mathrm{~Hz}), 1.33(3 \mathrm{H}, \mathrm{d}, J=6.4 \mathrm{~Hz}) ;{ }^{13} \mathrm{C}$ NMR $(125$ $\left.\mathrm{MHz} \mathrm{CDCl}_{3}\right): \delta 170.19,165.76,164.53,146.01,144.97,122.71,122.26,75.07,74.80$, $74.12,73.10,67.64,40.94,19.67,18.03,17.82 ;[\alpha]_{\mathrm{D}}{ }^{25}+81.0(c=0.195, \mathrm{MeOH})$ [lit. $^{{ }^{\mathrm{b}}}$ $\left.[\alpha]_{\mathrm{D}}^{23}+84.1(c=0.59, \mathrm{MeOH})\right]$.

Macrosphelide B (2). Pyridinium dichromate (137 $\mathrm{mg}, 0.37 \mathrm{mmol})$ was added portionwise to a stirred solution of the alcohol $18(39.3 \mathrm{mg}, 0.091 \mathrm{mmol})$ and molecular sieves $4 \mathrm{~A}(150 \mathrm{mg})$ in $\mathrm{CH}_{2} \mathrm{Cl}_{2}(10 \mathrm{ml})$ at $0{ }^{\circ} \mathrm{C}$ under $\mathrm{Ar}$ atmosphere. After continuous stirring for $1 \mathrm{~h}$ at room temperature, the reaction mixture was diluted with ether $(20 \mathrm{ml})$, 
and filtered through celite. The filtrate was evaporated to leave a residue which was chromatographed on silica gel with hexane-EtOAc $(1: 1)$ to give the corresponding ketone $(32.0 \mathrm{mg}, 82 \%)$ as a colorless oil. ${ }^{1} \mathrm{H}$ NMR $\left(300 \mathrm{MHz}, \mathrm{CDCl}_{3}\right): \delta 6.98(1 \mathrm{H}, \mathrm{d}$, $J=16 \mathrm{~Hz}), 6.82(1 \mathrm{H}, \mathrm{dd}, J=16,6.0 \mathrm{~Hz}), 6.73(1 \mathrm{H}, \mathrm{d}, J=16 \mathrm{~Hz}), 6.04(1 \mathrm{H}, \mathrm{dd}, J=16,1.4$ $\mathrm{Hz}), 5.39-5.35(1 \mathrm{H}, \mathrm{m}), 5.17(1 \mathrm{H}, \mathrm{dd}, J=14,7.0 \mathrm{~Hz}), 5.04-5.02(1 \mathrm{H}, \mathrm{m}), 4.76(1 \mathrm{H}, \mathrm{d}$, $J=7.1 \mathrm{~Hz}), 4.73(1 \mathrm{H}, \mathrm{d}, J=7.1 \mathrm{~Hz}), 4.27-4.24(1 \mathrm{H}, \mathrm{m}), 3.80-3.74(1 \mathrm{H}, \mathrm{m}), 3.70-3.63$ $(1 \mathrm{H}, \mathrm{m}), 3.58-3.52(2 \mathrm{H}, \mathrm{m}), 3.38(3 \mathrm{H}, \mathrm{s}), 2.75(1 \mathrm{H}, \mathrm{dd}, J=16,10 \mathrm{~Hz}), 2.62(1 \mathrm{H}, \mathrm{dd}$, $J=16,2.6 \mathrm{~Hz}), 1.43(3 \mathrm{H}, \mathrm{d}, J=6.3 \mathrm{~Hz}), 1.41(3 \mathrm{H}, \mathrm{d}, J=6.9 \mathrm{~Hz}), 1.36(3 \mathrm{H}, \mathrm{d}, J=6.3 \mathrm{~Hz})$; ${ }^{13} \mathrm{C}$ NMR $\left(75 \mathrm{MHz}, \mathrm{CDCl}_{3}\right): \delta 195.85,170.25,164.33,163.73,143.85,133.13,132.41$, 124.17, 94.21, 78.27, 75.50, 72.49, 71.70, 68.33, 67.61, 59.23, 41.14, 20.12, 17.95, 16.12; IR (neat): 1729, $1660 \mathrm{~cm}^{-1}$; MS (EI) $\mathrm{m} / z 428\left(\mathrm{M}^{+}\right)$; HRMS (EI) Calcd for $\mathrm{C}_{20} \mathrm{H}_{28} \mathrm{O}_{10}: 428.1683\left(\mathrm{M}^{+}\right)$, found: 428.1688; $[\alpha]_{\mathrm{D}}{ }^{24}-74.9\left(c=1.43, \mathrm{CHCl}_{3}\right)$.

Trifluoroacetic acid $(1.5 \mathrm{ml})$ was added to a solution of the ketone $(52.3 \mathrm{mg}, 0.122$ mmol $)$ in $\mathrm{CH}_{2} \mathrm{Cl}_{2}(1.5 \mathrm{ml})$ at $0{ }^{\circ} \mathrm{C}$ under Ar atmosphere. After continuous stirring for 24 $\mathrm{h}$ at room temperature, the solvent was evaporated to afford a residue which was chromatographed on silica gel with hexane-EtOAc $(1: 1)$ to give macrosphelide $\mathrm{B}(2)$ (35.7 mg, 86\%) as a colorless oil. ${ }^{1} \mathrm{H}$ NMR $\left(300 \mathrm{MHz}, \mathrm{CDCl}_{3}\right): \delta 7.02(1 \mathrm{H}, \mathrm{d}, J=16 \mathrm{~Hz})$, $6.92(1 \mathrm{H}, \mathrm{dd}, J=16,3.7 \mathrm{~Hz}), 6.73(1 \mathrm{H}, \mathrm{d}, J=16 \mathrm{~Hz}), 6.07(1 \mathrm{H}, \mathrm{dd}, J=16,1.8 \mathrm{~Hz}), 5.47-$ $5.44(1 \mathrm{H}, \mathrm{m}), 5.10-5.00(2 \mathrm{H}, \mathrm{m}), 4.32(1 \mathrm{H}, \mathrm{brs}), 3.10(1 \mathrm{H}, \mathrm{brs}), 2.83(1 \mathrm{H}, \mathrm{dd}, J=16,11$ $\mathrm{Hz}), 2.62(1 \mathrm{H}, \mathrm{dd}, J=16,2.2 \mathrm{~Hz}), 1.49(3 \mathrm{H}, \mathrm{d}, J=6.9 \mathrm{~Hz}), 1.43(3 \mathrm{H}, \mathrm{d}, J=7.1 \mathrm{~Hz}), 1.35$ $(3 \mathrm{H}, \mathrm{d}, J=6.3 \mathrm{~Hz}) ;{ }^{13} \mathrm{C} \mathrm{NMR}\left(125 \mathrm{MHz} \mathrm{CDCl}_{3}\right): \delta 196.18,170.33,165.46,164.12$, 144.15, 132.51, 132.01, 122.55, 76.92, 75.80, 74.86, 67.66, 40.58, 19.79, 17.94, 16.09; $[\alpha]_{\mathrm{D}}{ }^{27}+3.71(c=0.335, \mathrm{MeOH})\left[\right.$ lit. $\left.^{\mathrm{b}]}[\alpha]_{\mathrm{D}}{ }^{23}+4.10(c=0.99, \mathrm{MeOH})\right]$. 


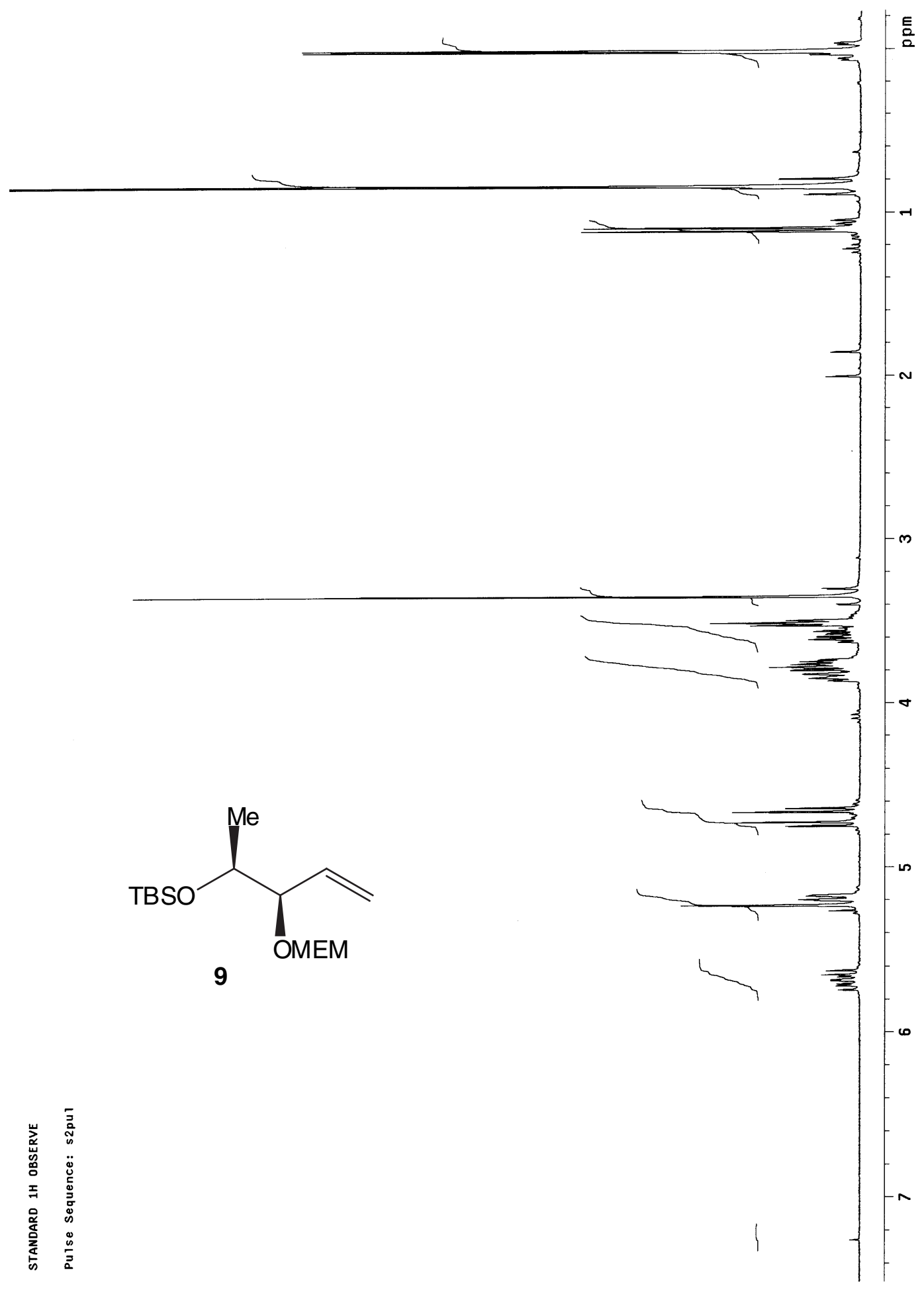




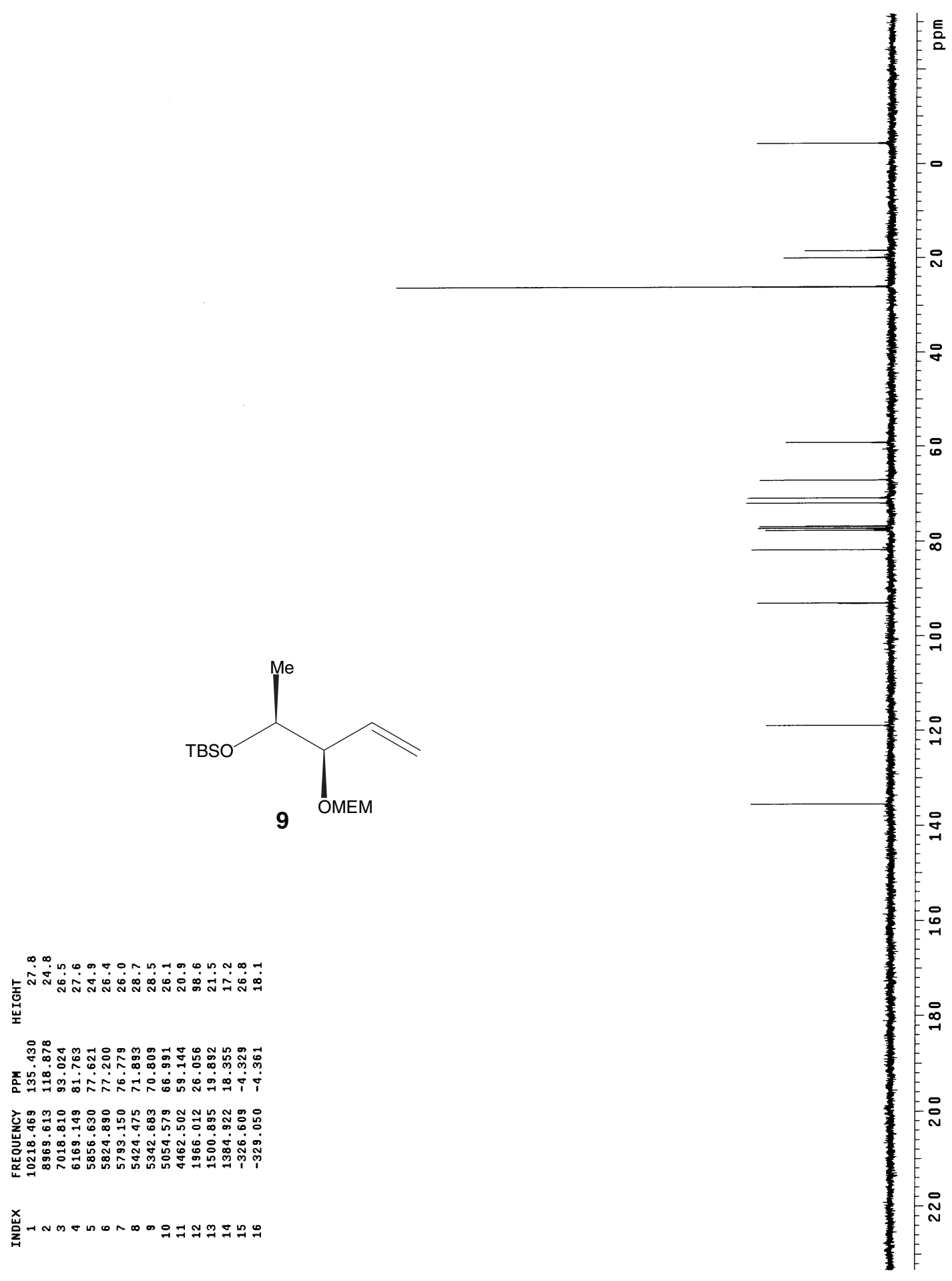




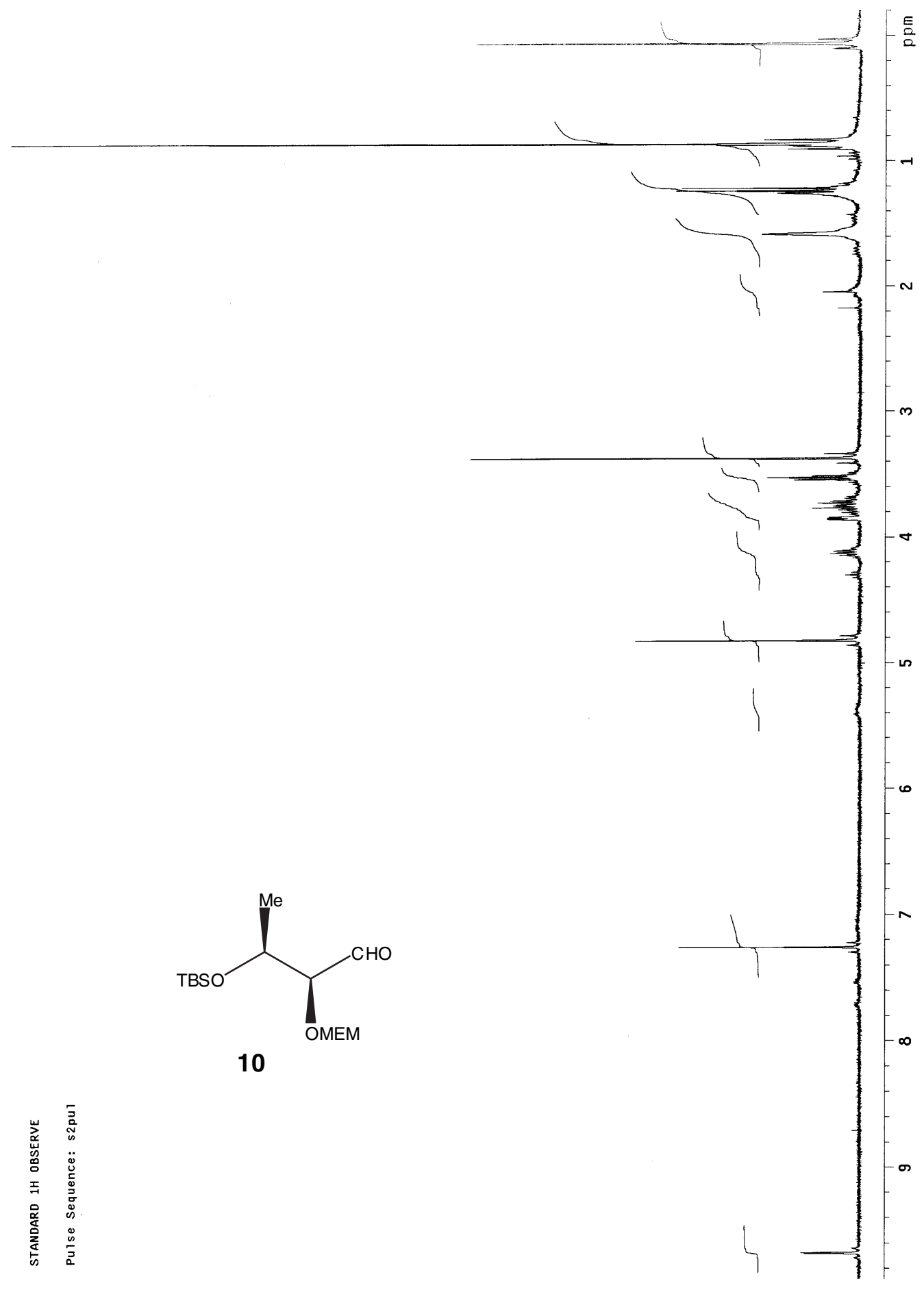



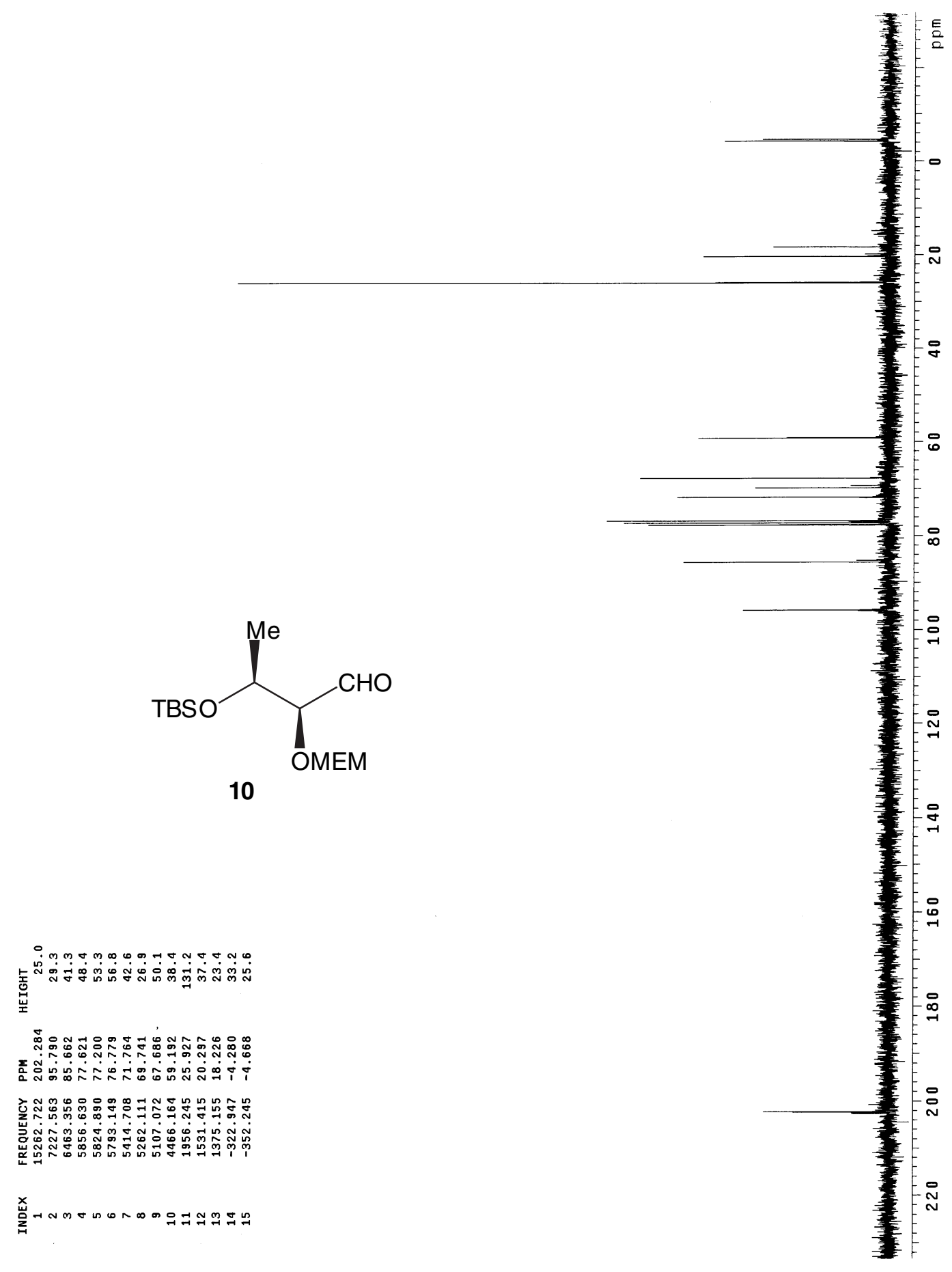


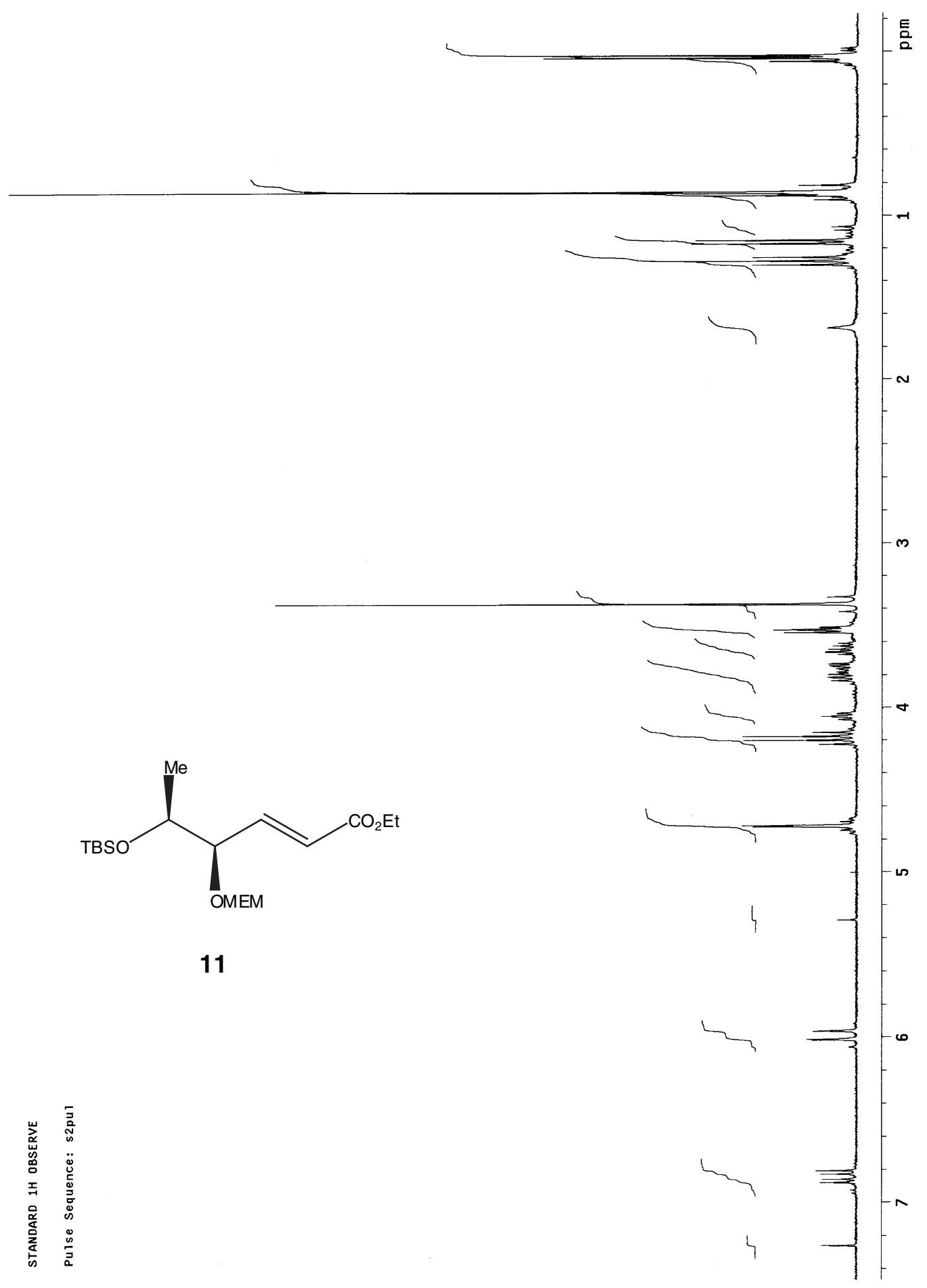



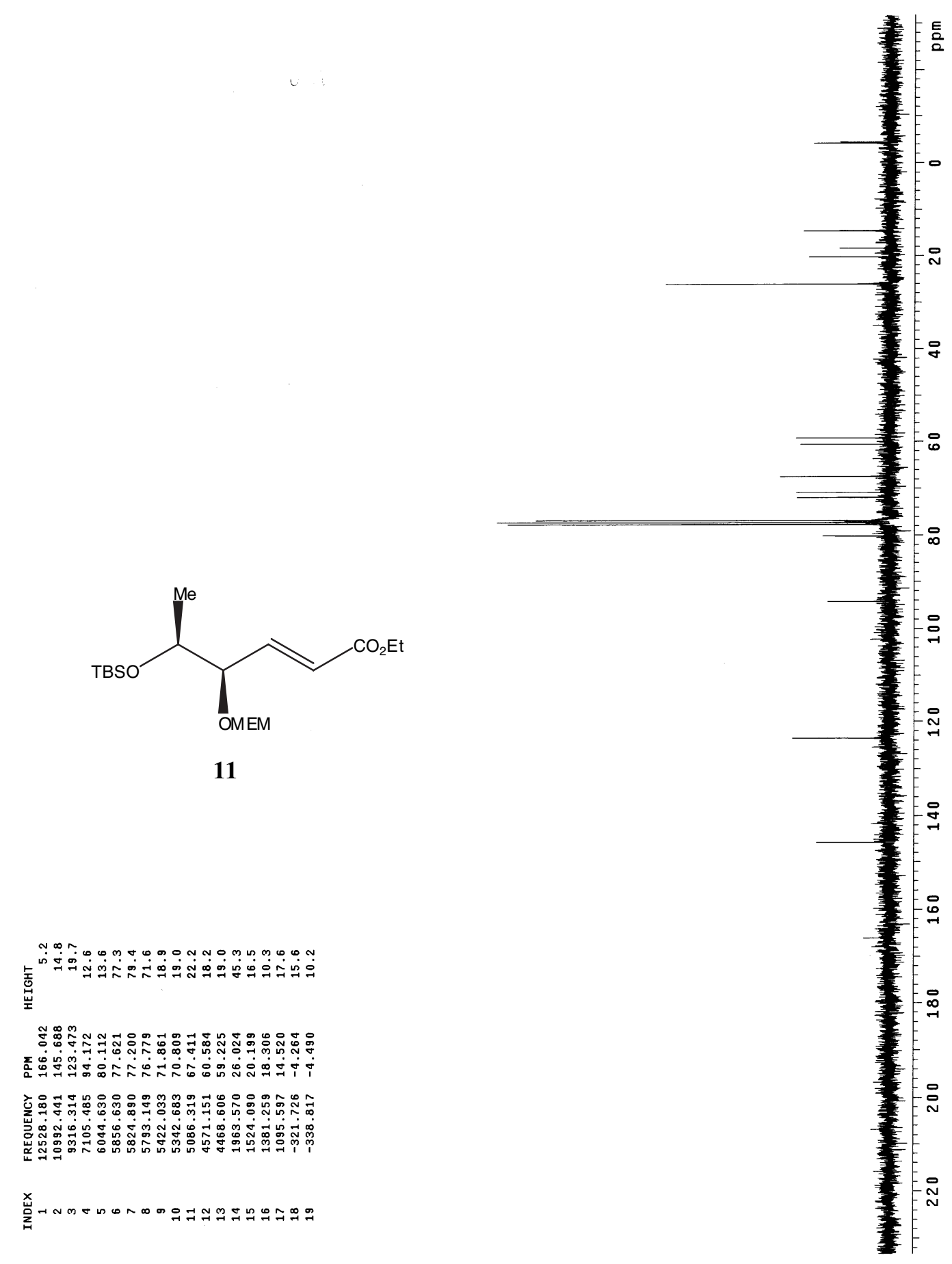


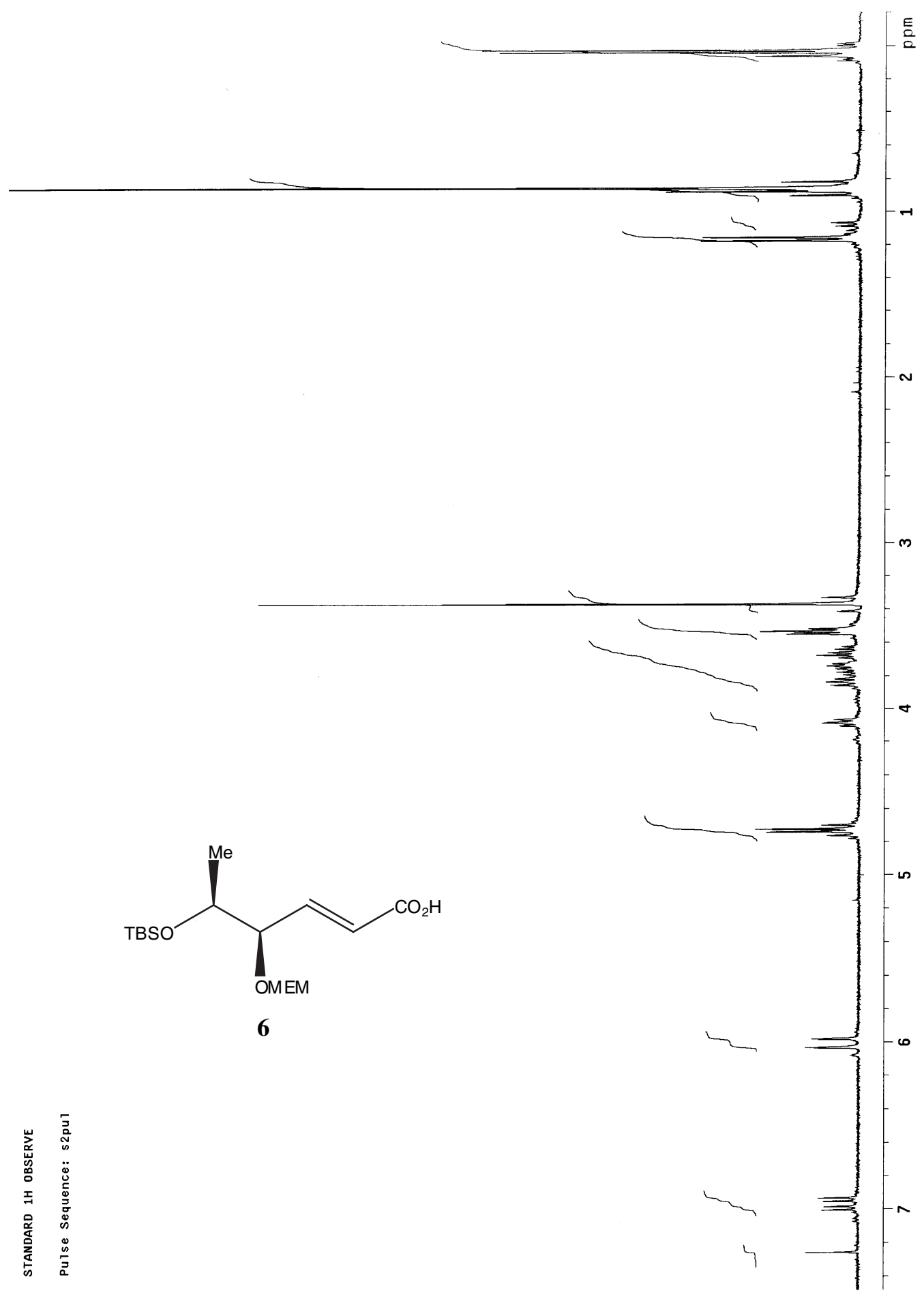



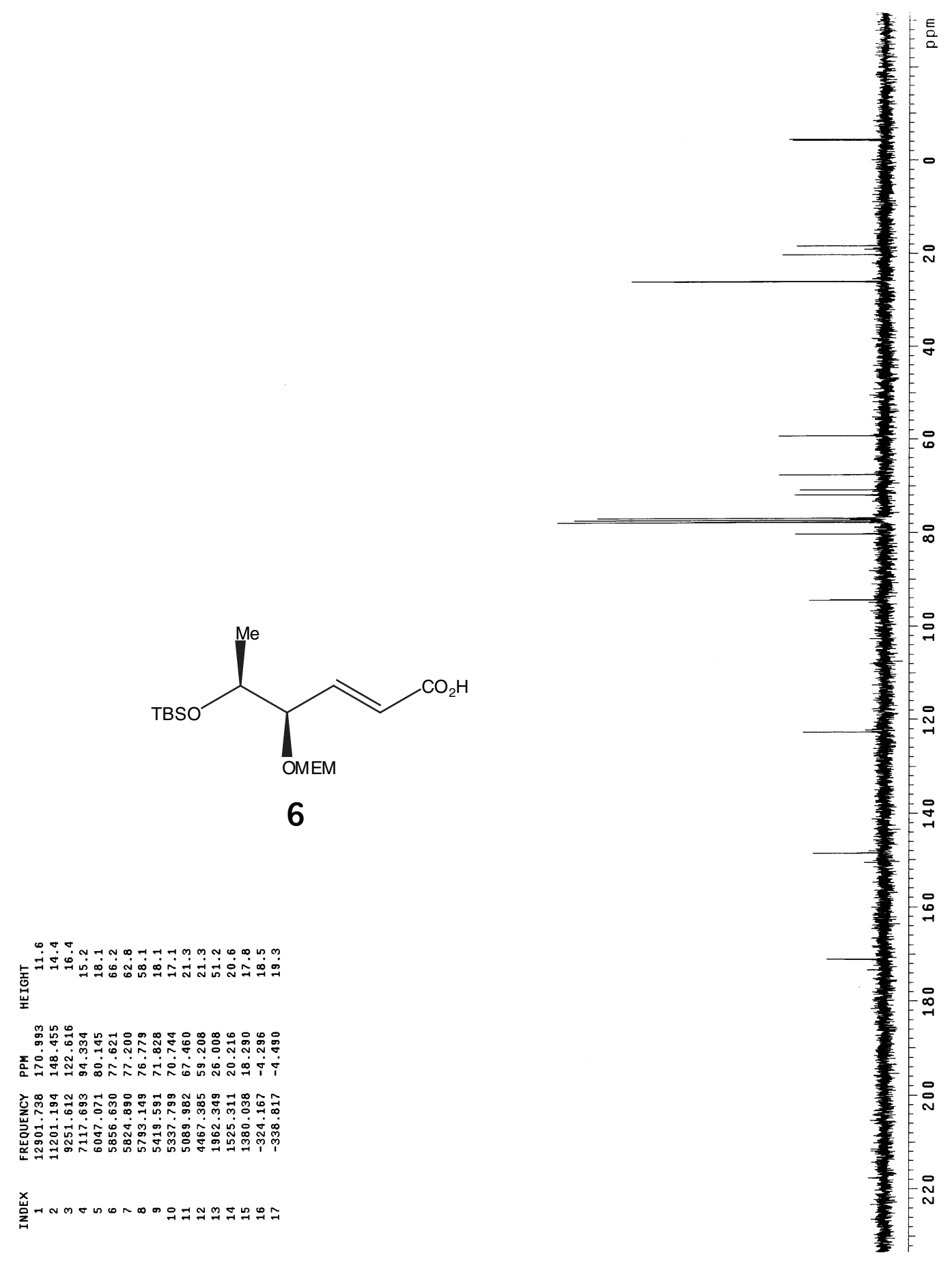


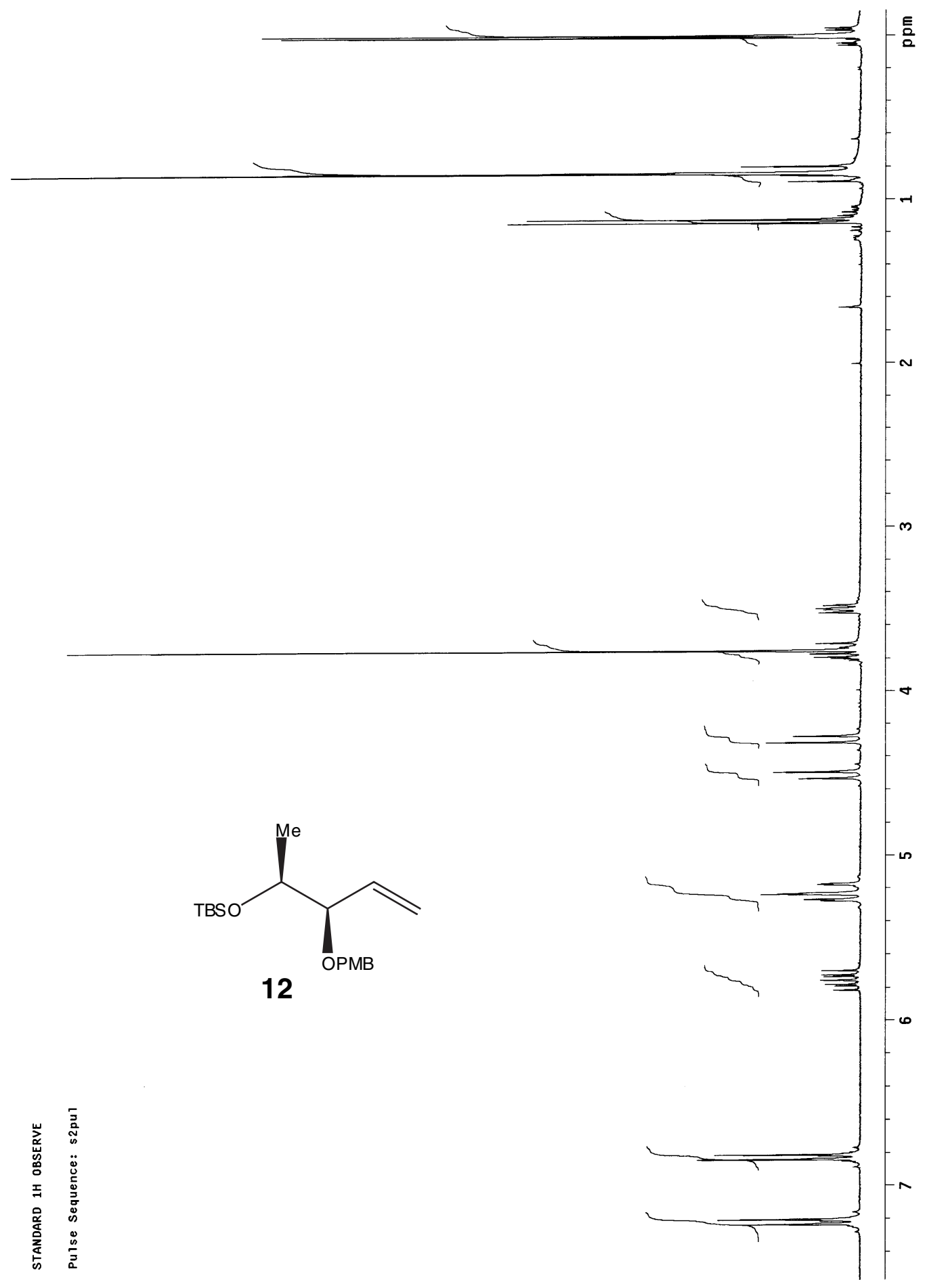



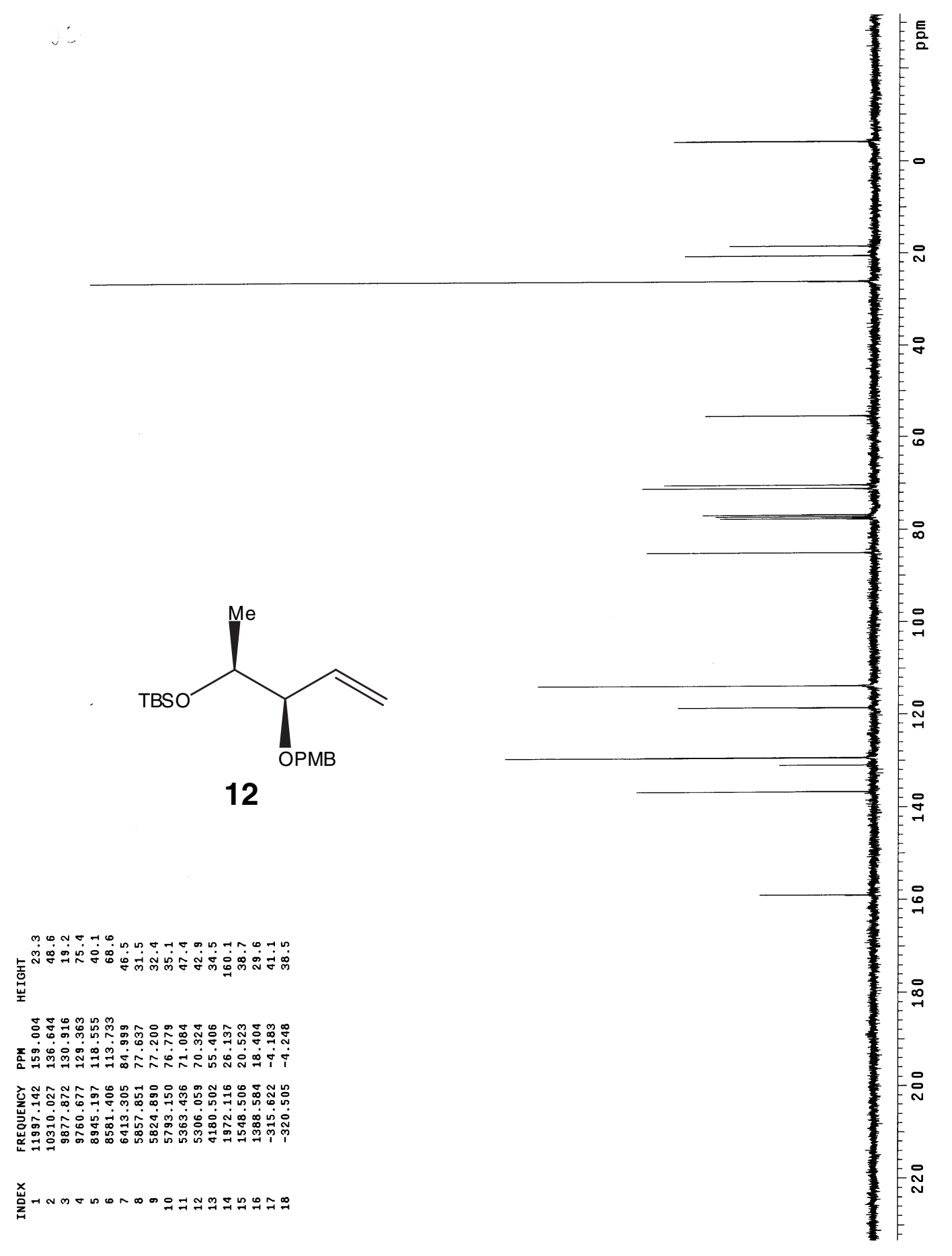


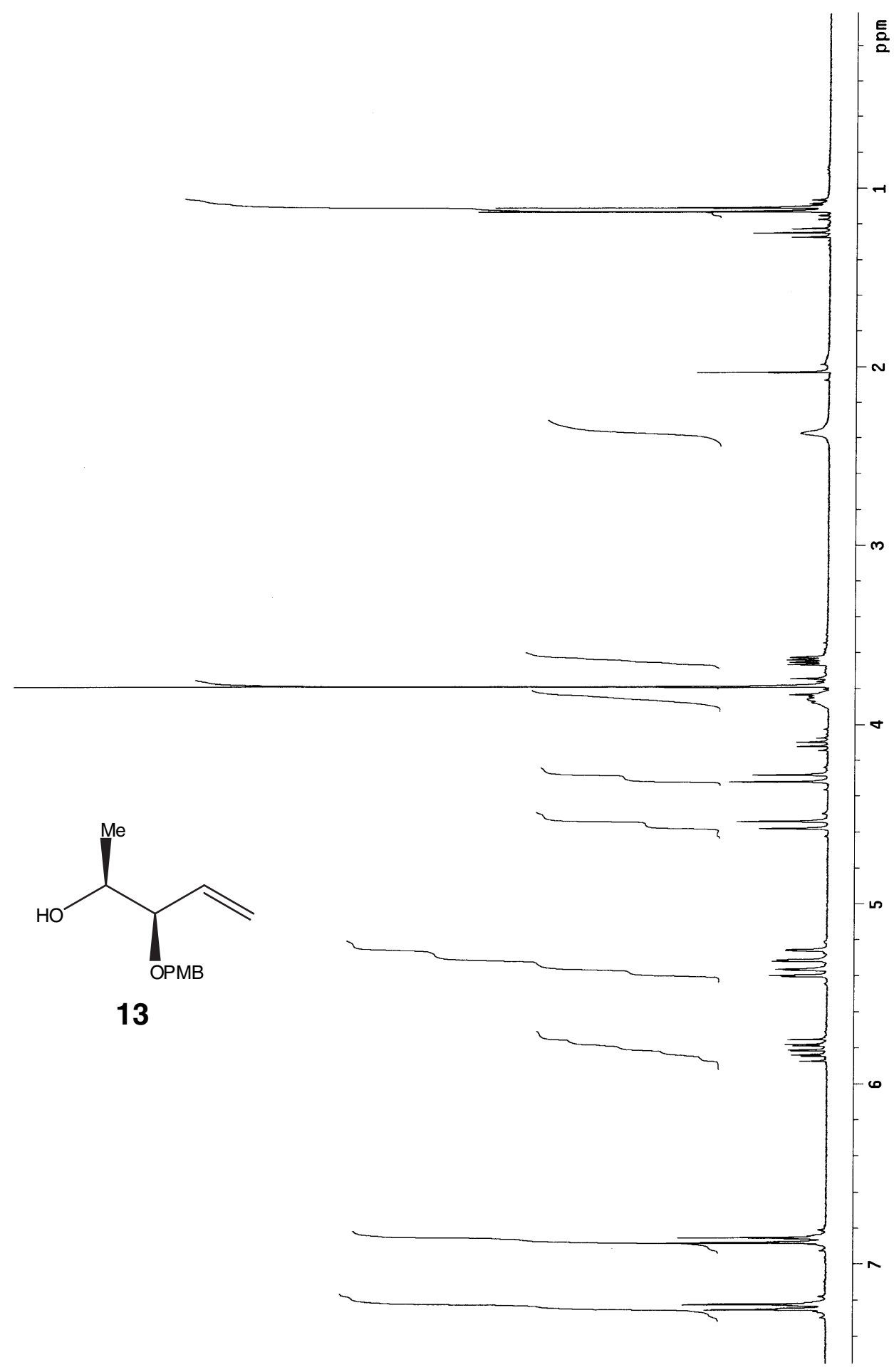




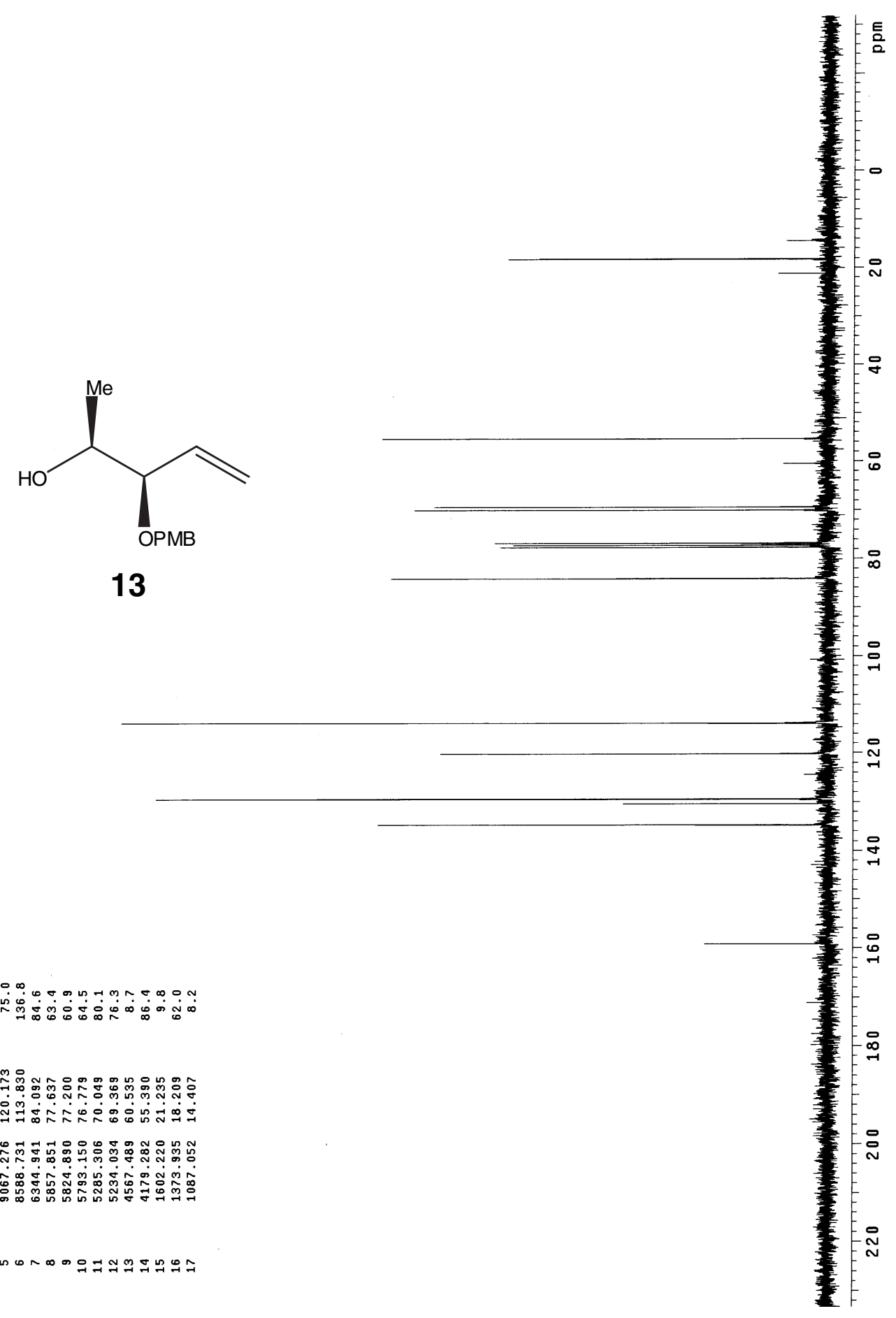




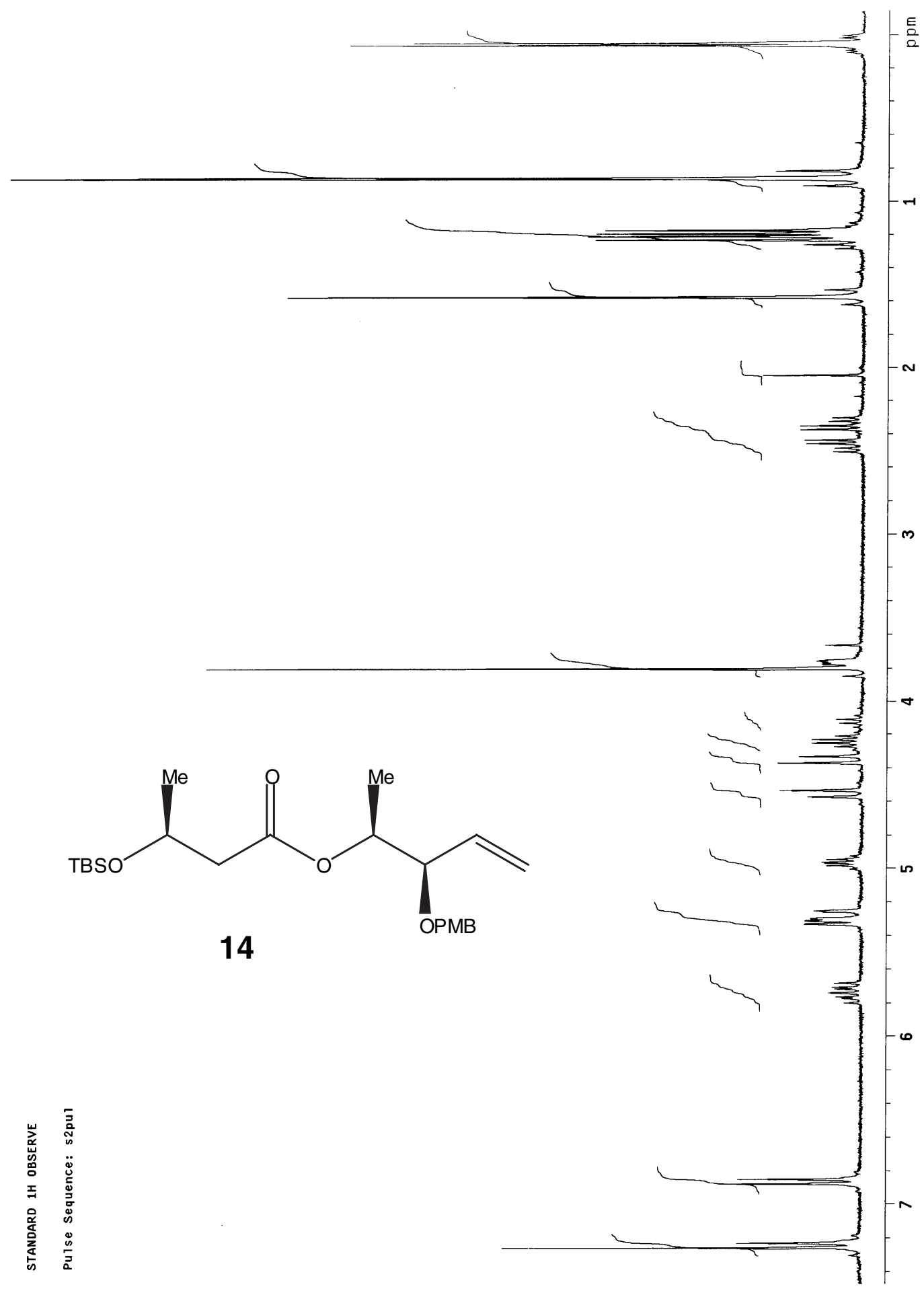




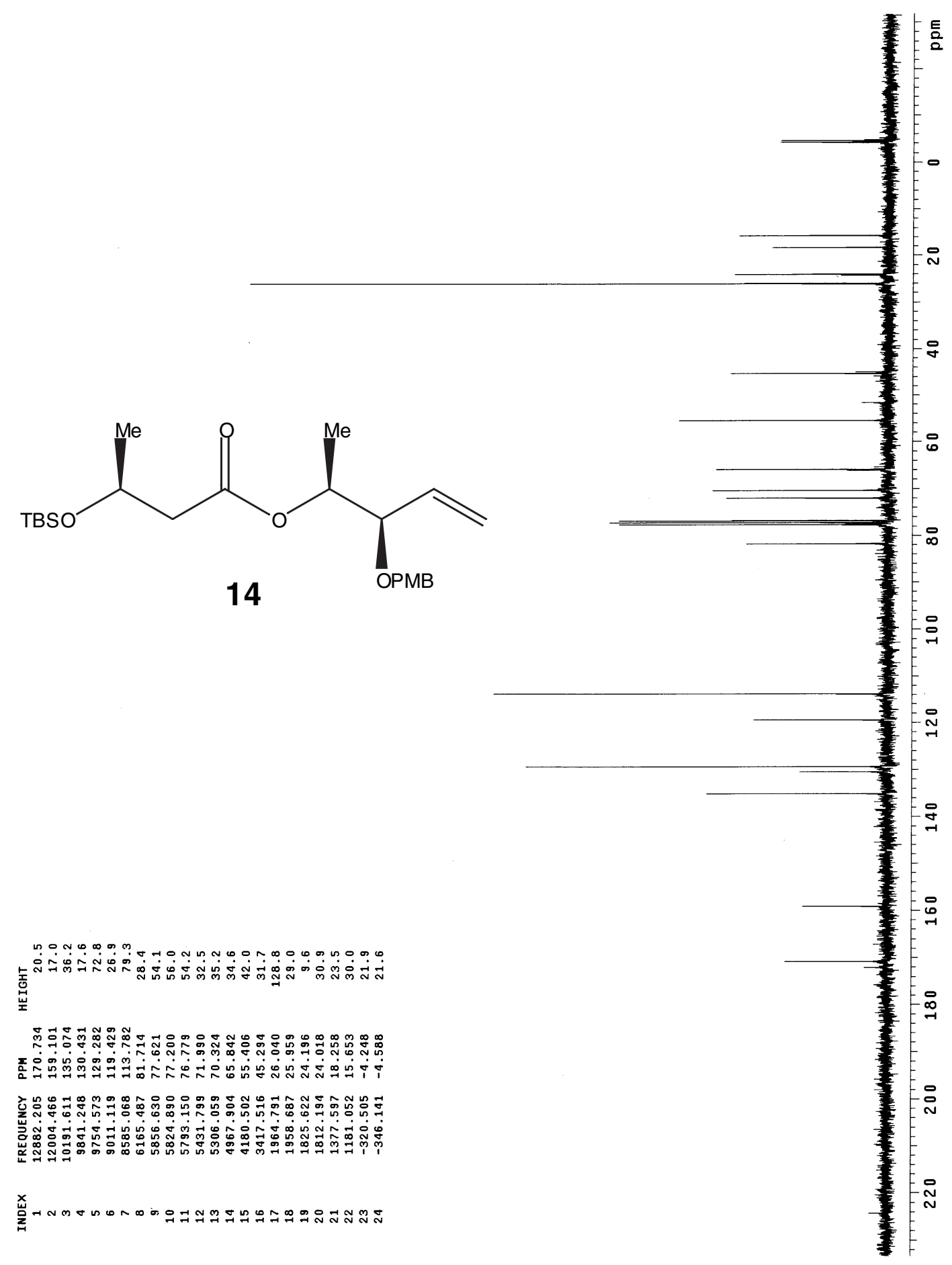




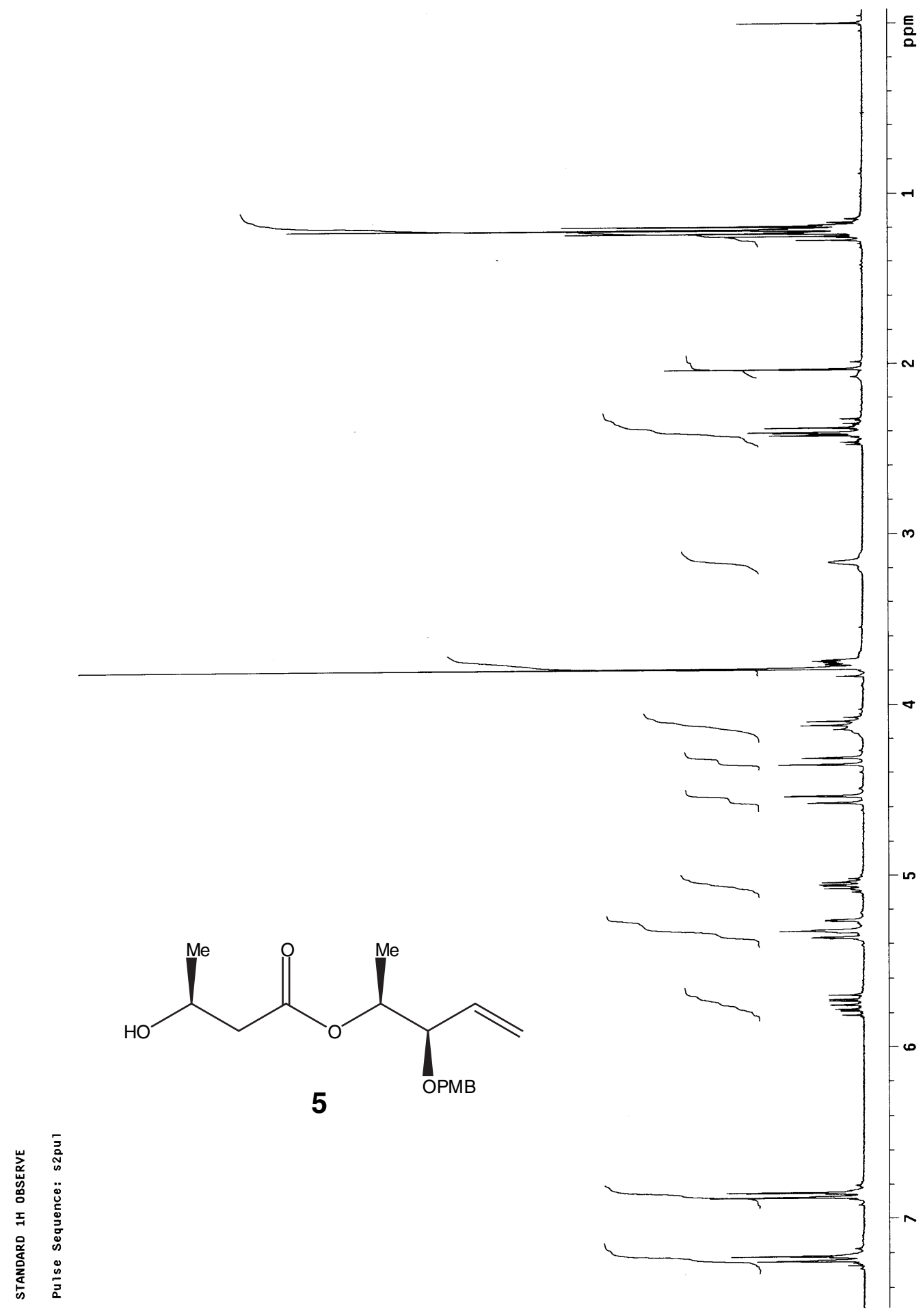




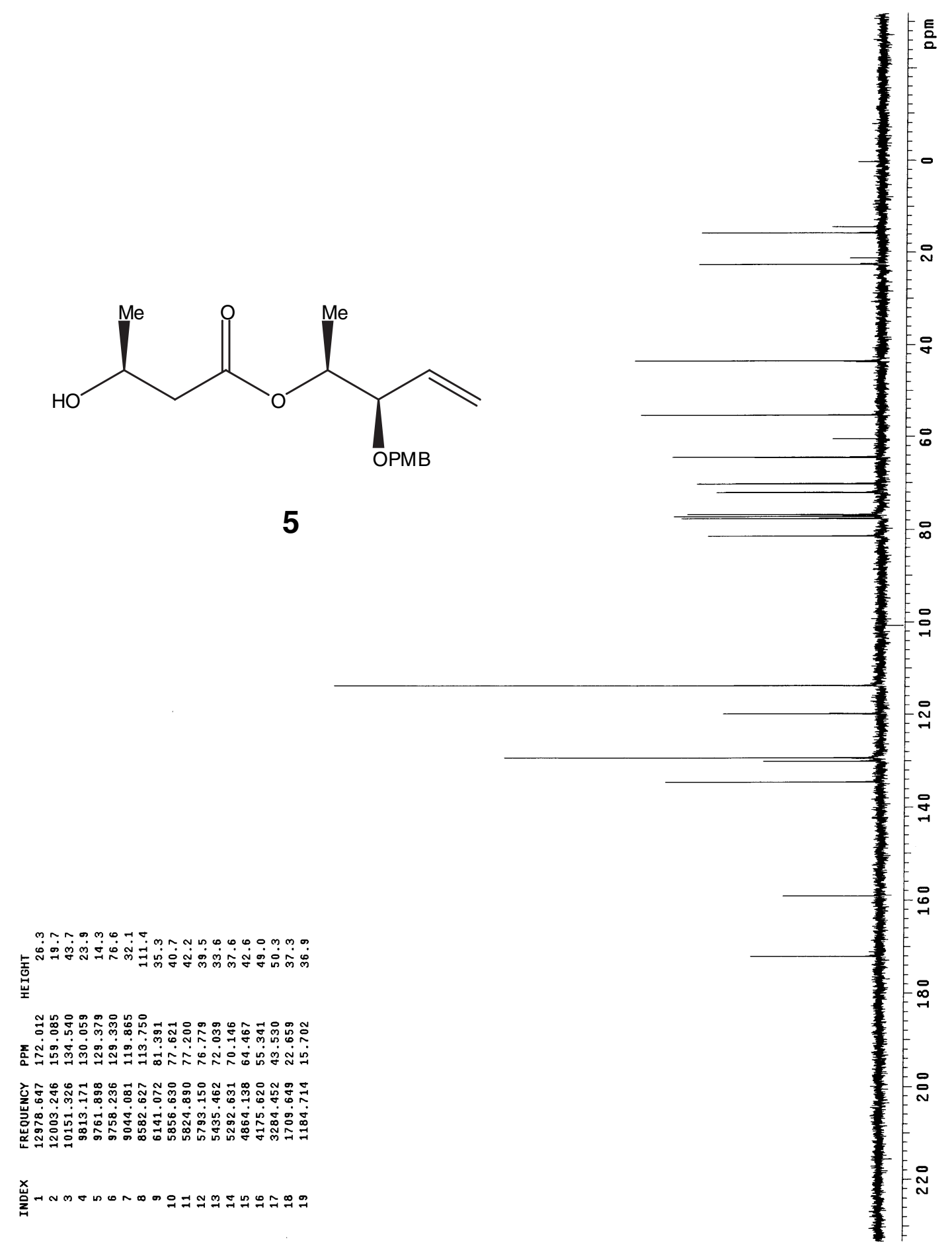




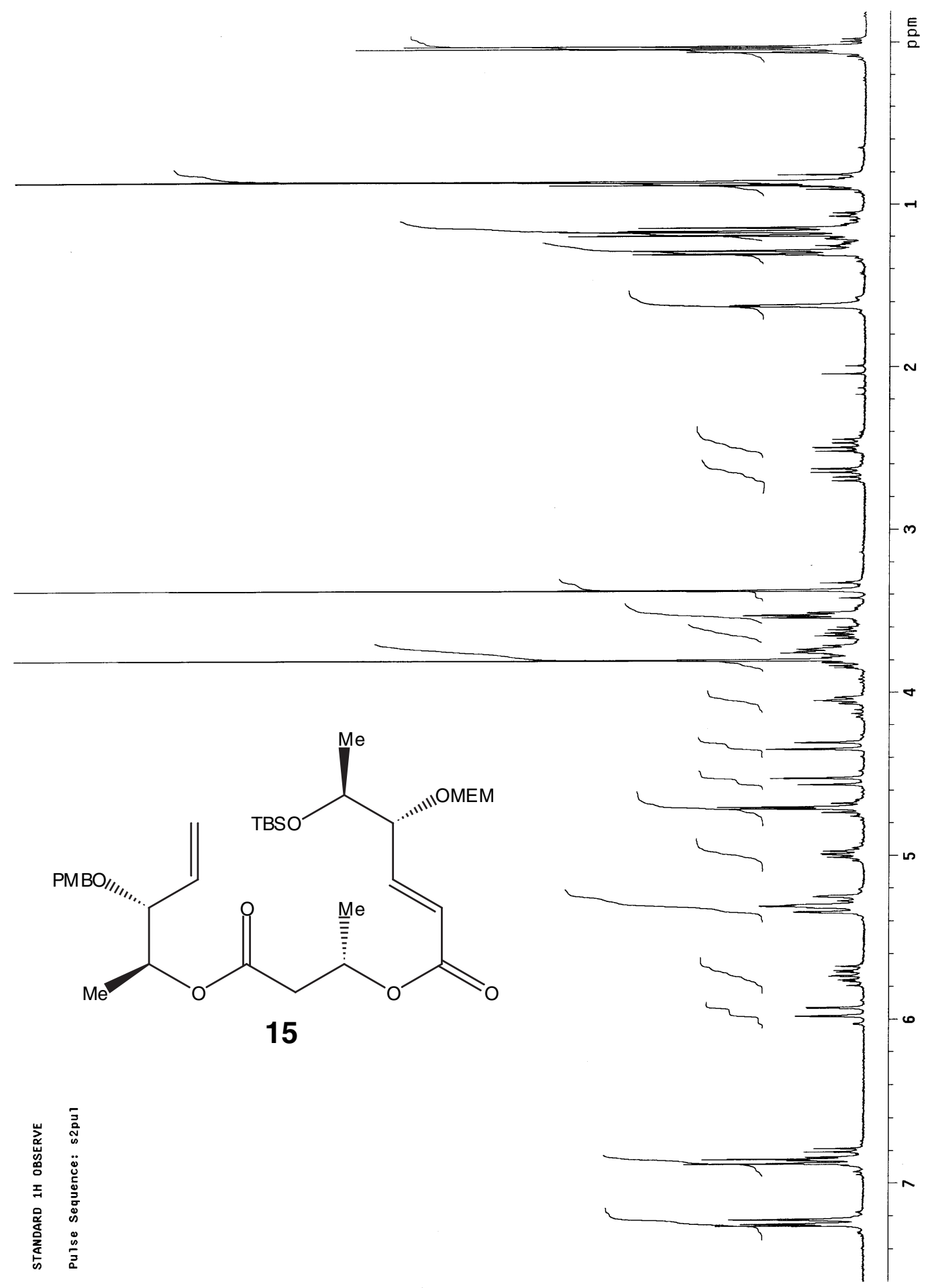




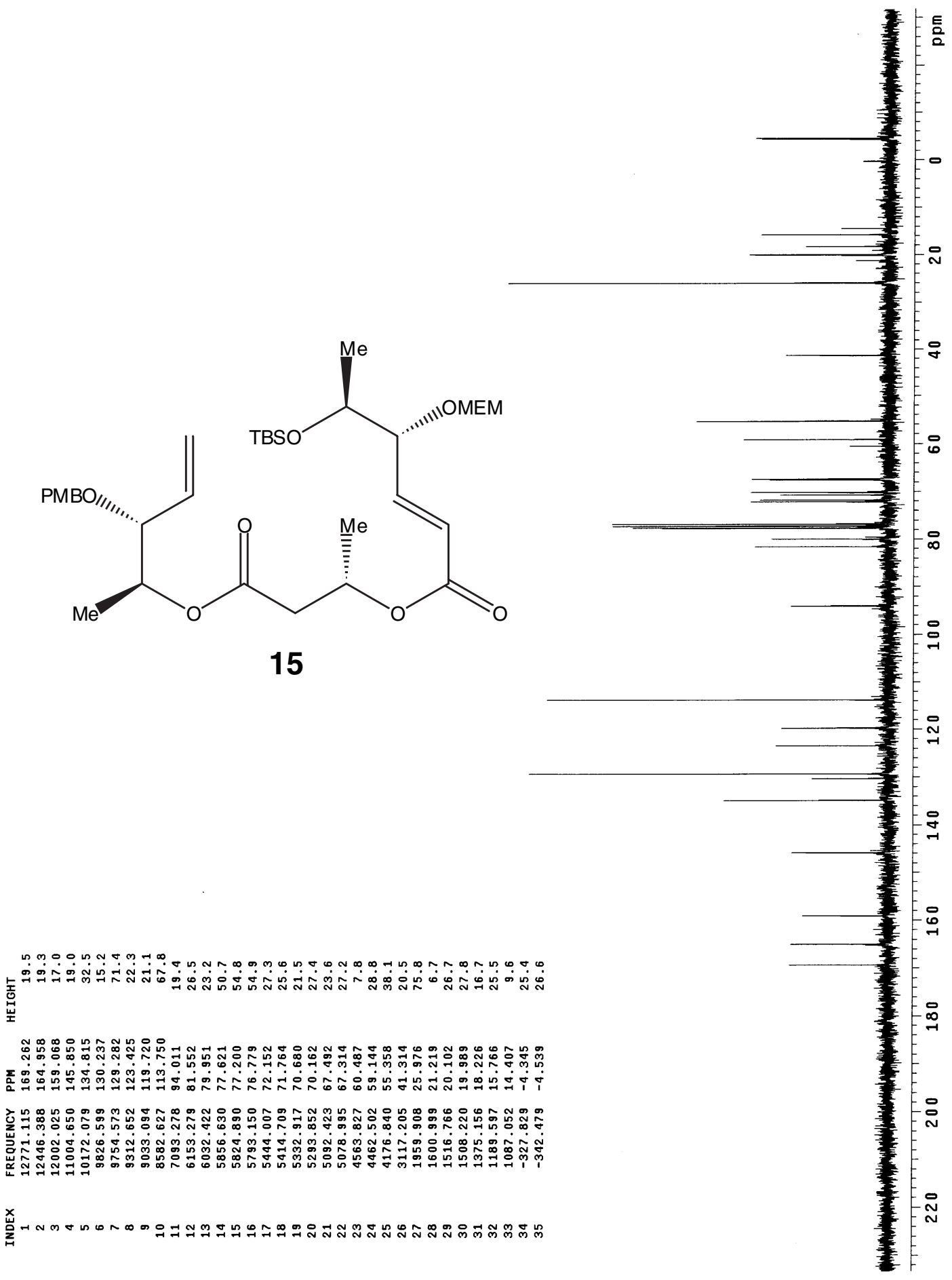




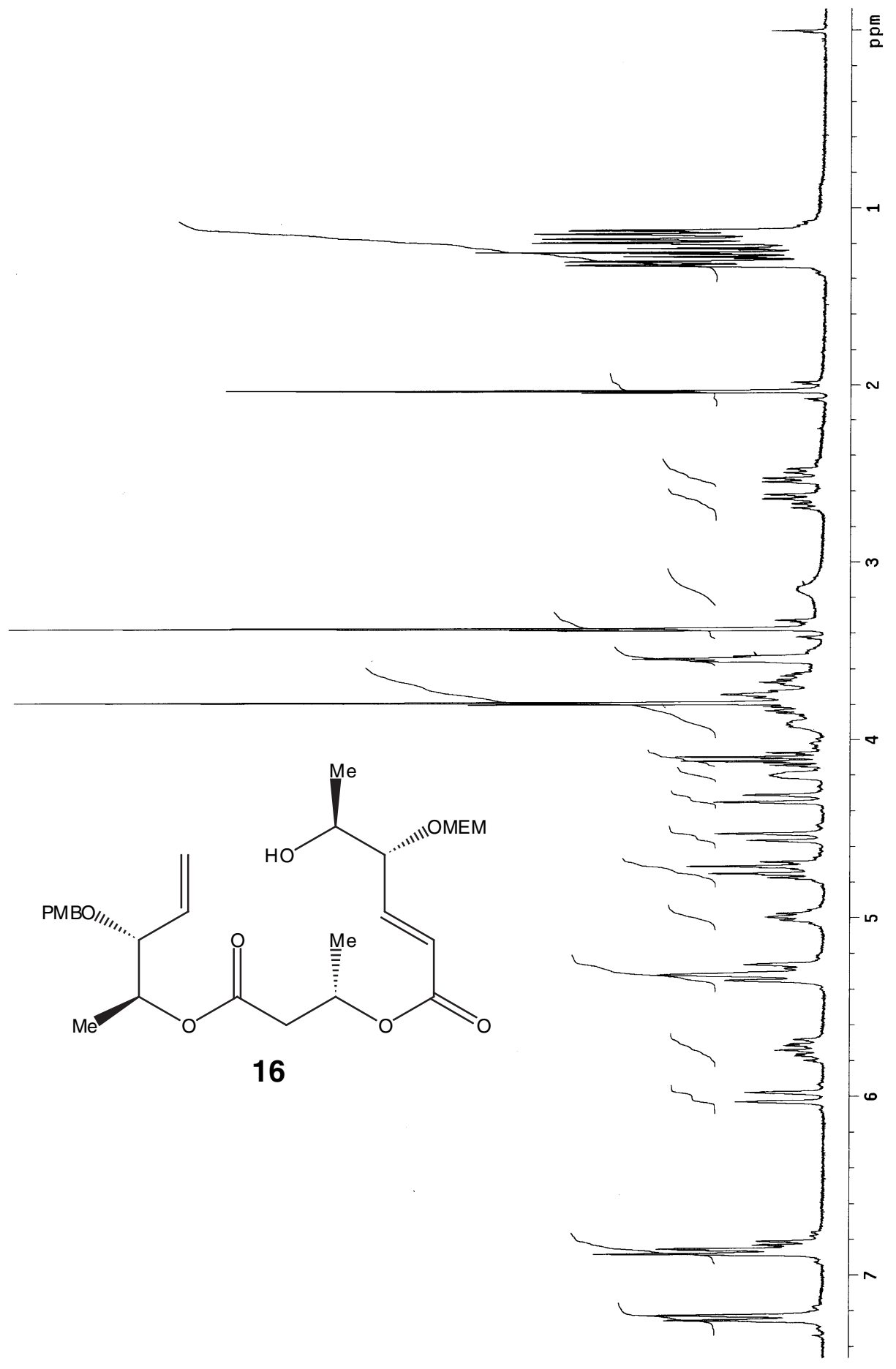




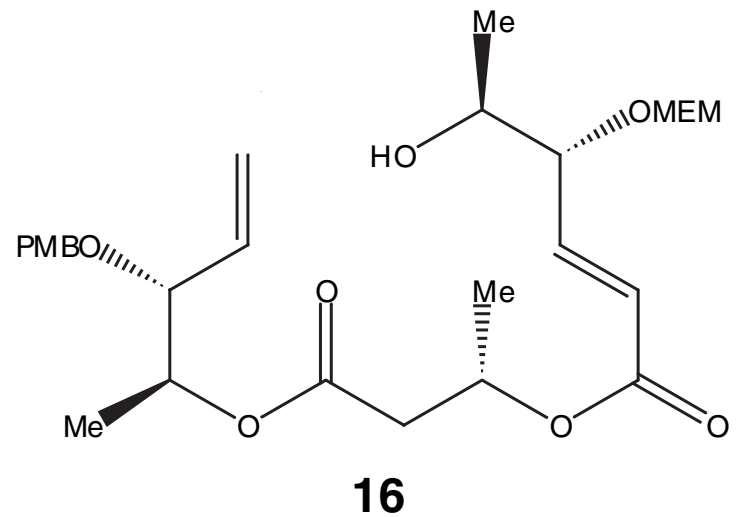

16

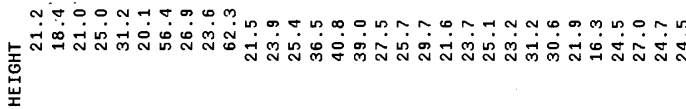

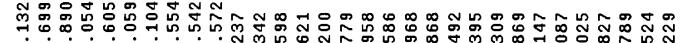

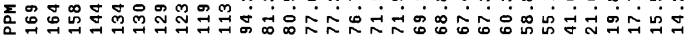

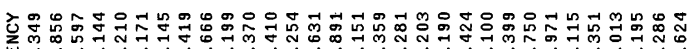

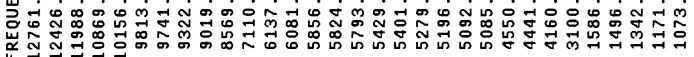

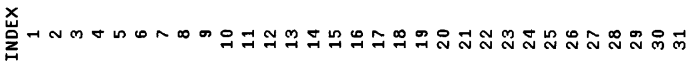

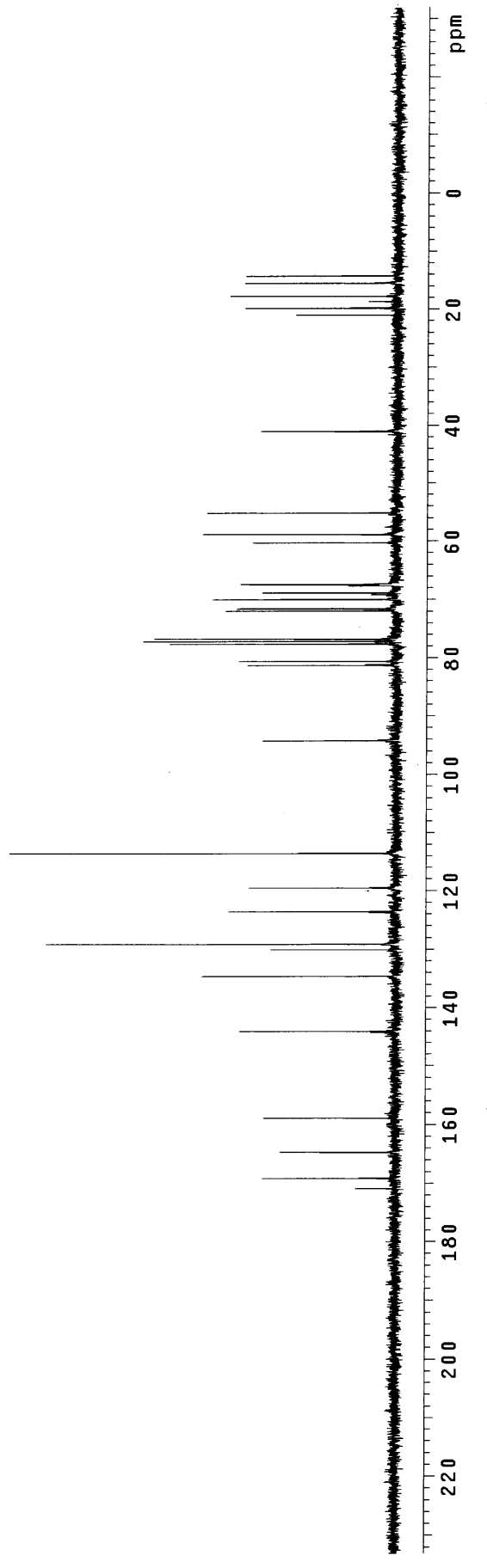




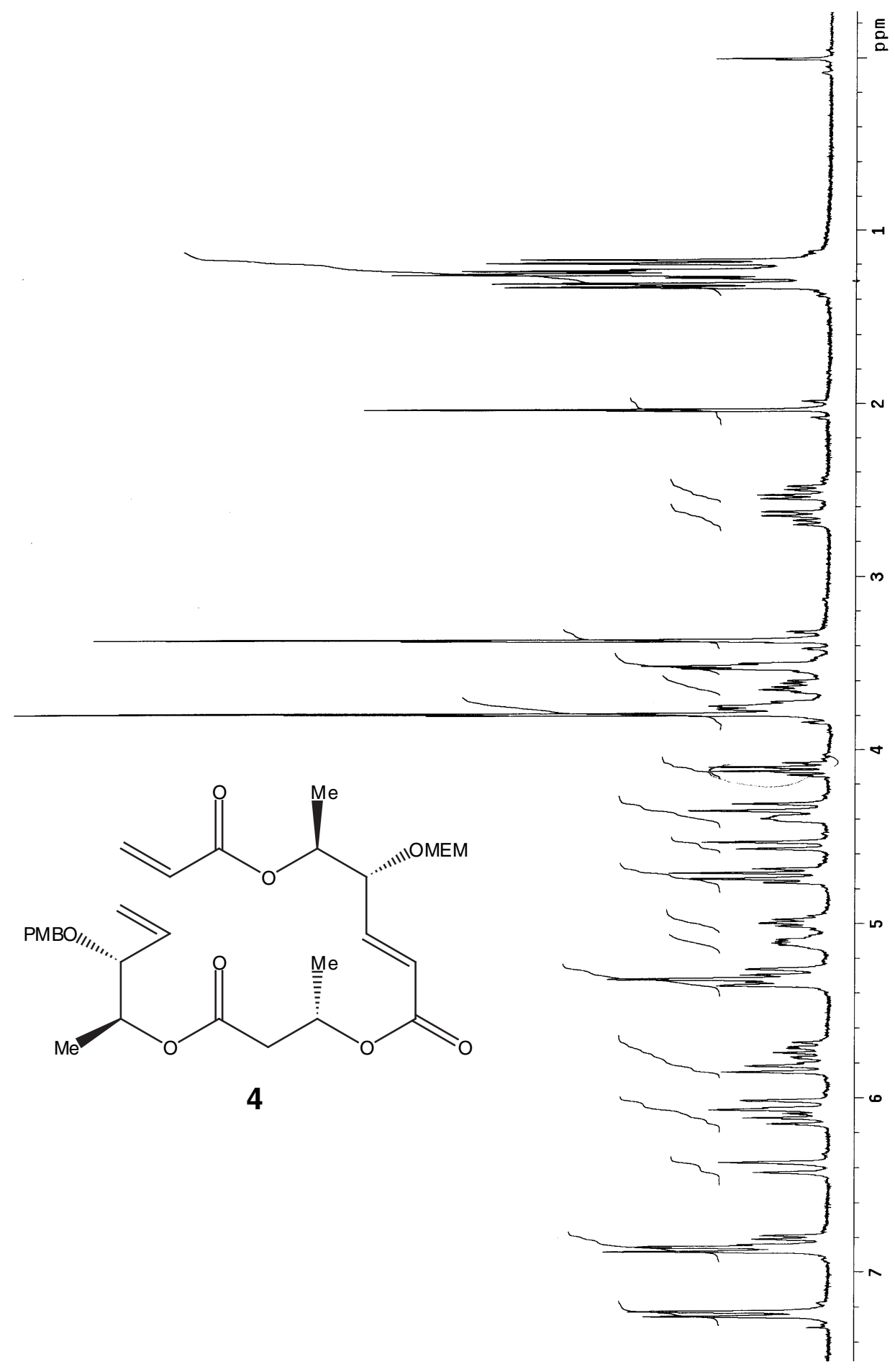



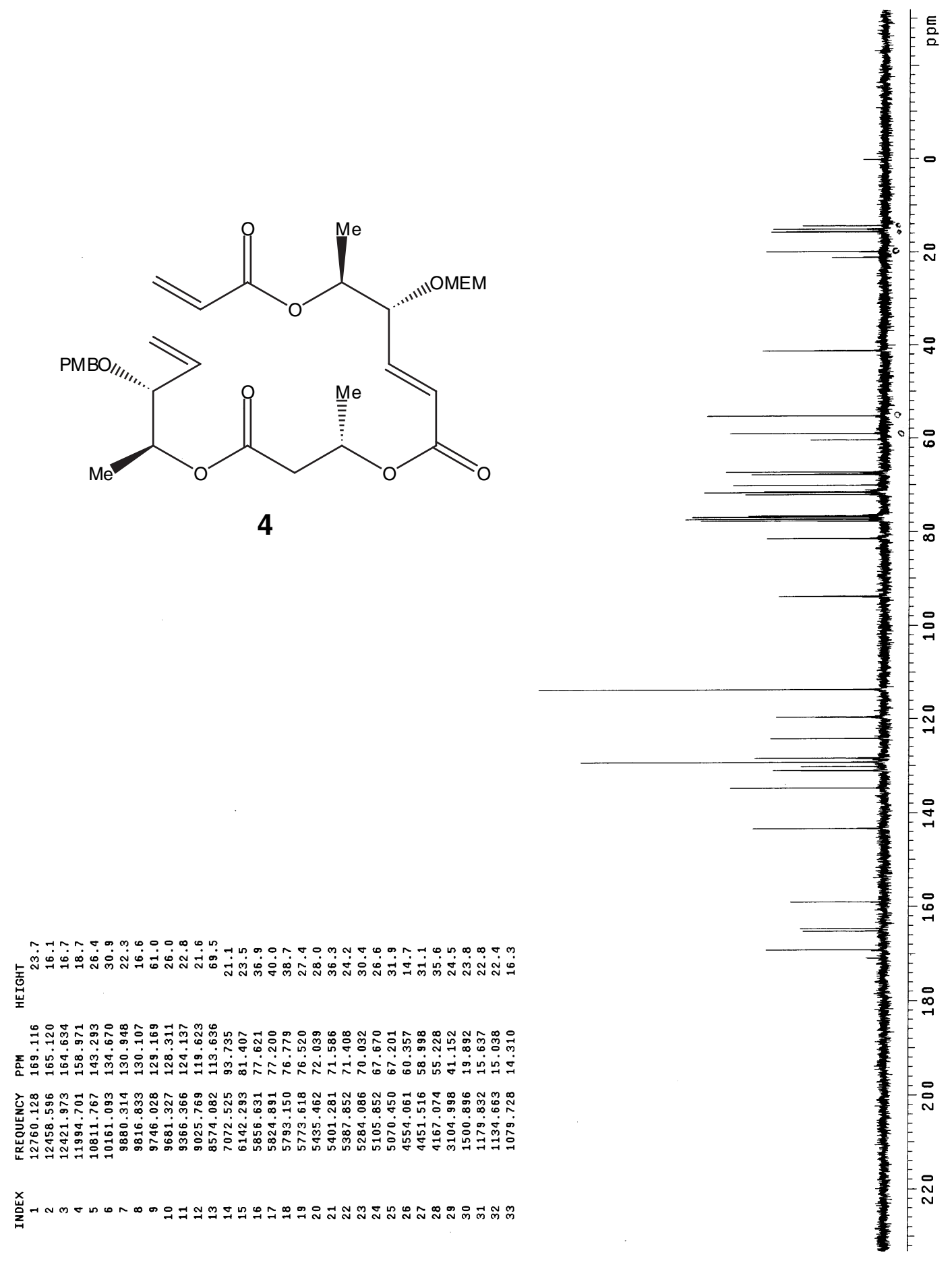


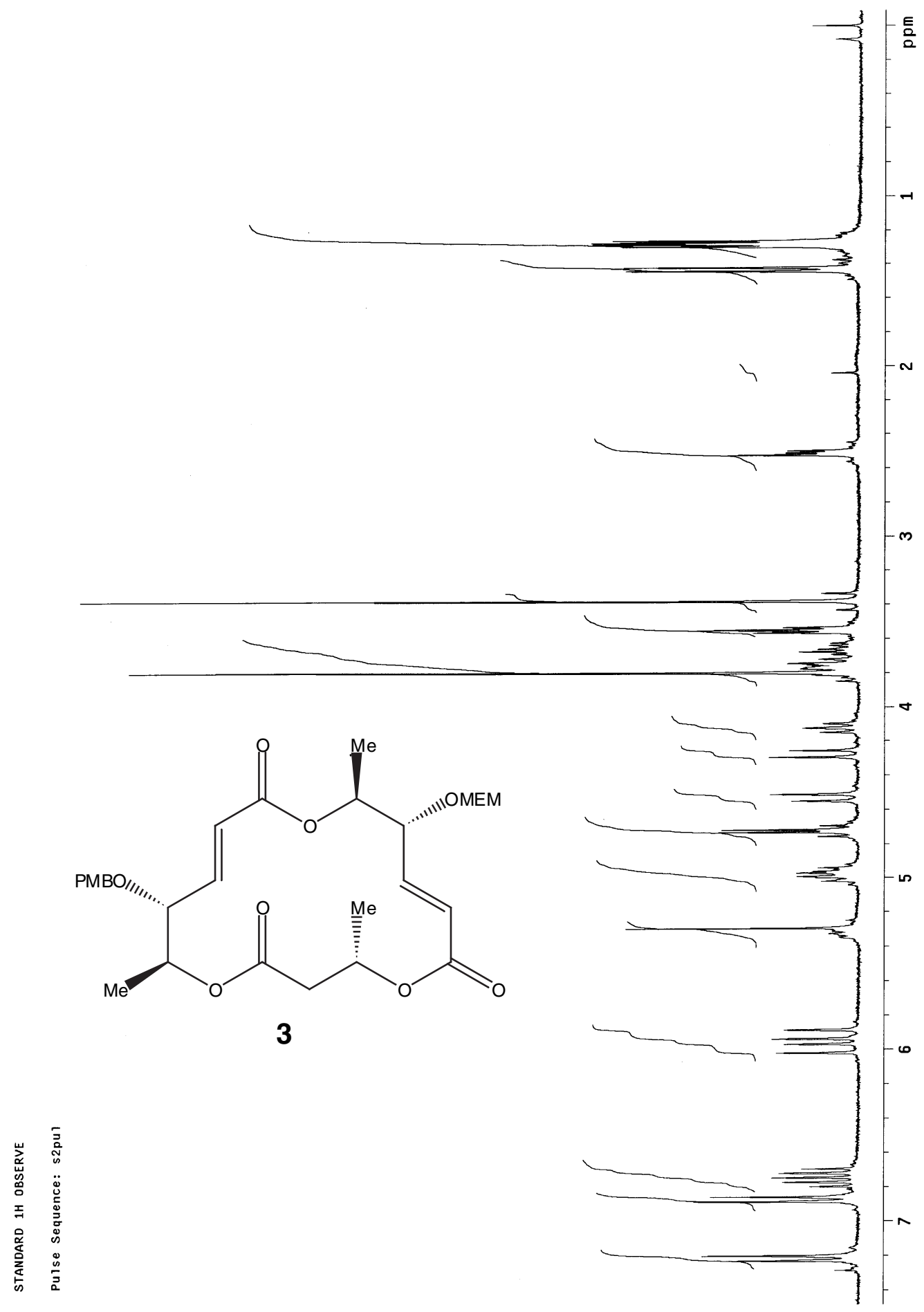


<smiles>CCCC[C@@H](C)OC(=O)/C=C/[C@@H](OC)[C@@H](C)OC(=O)C[C@@H](C)OC(=O)/C=C/[C@@H](OC)[C@@H](C)OC</smiles>

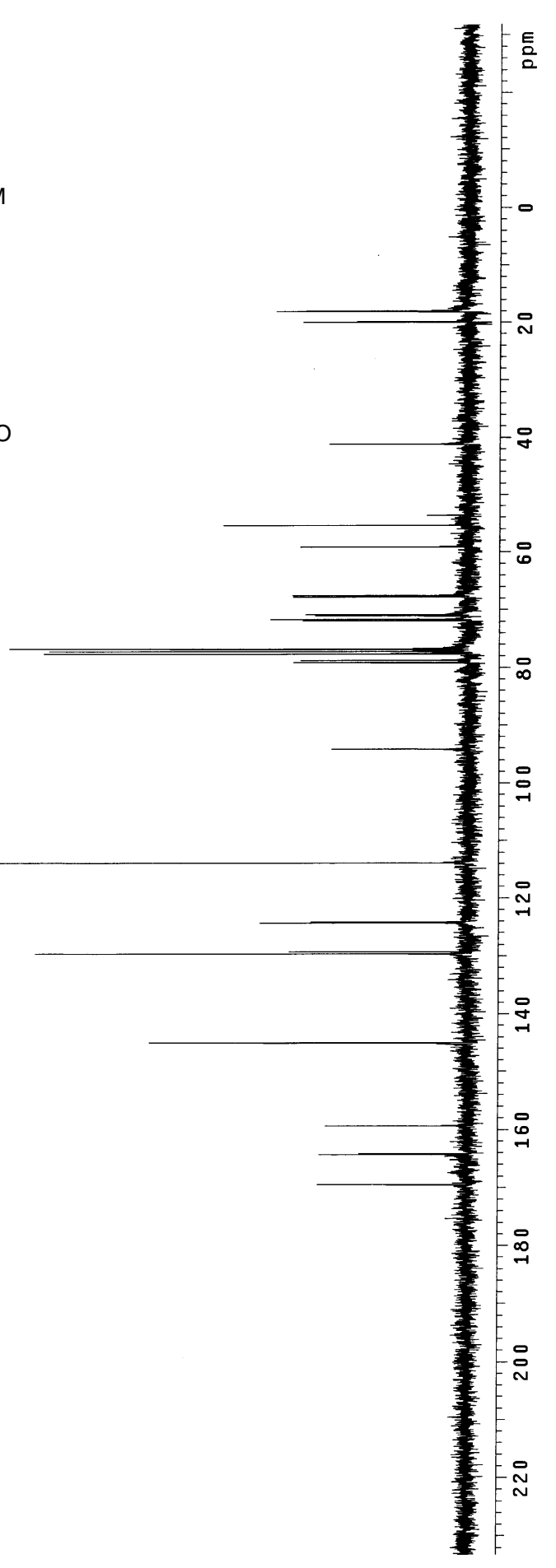




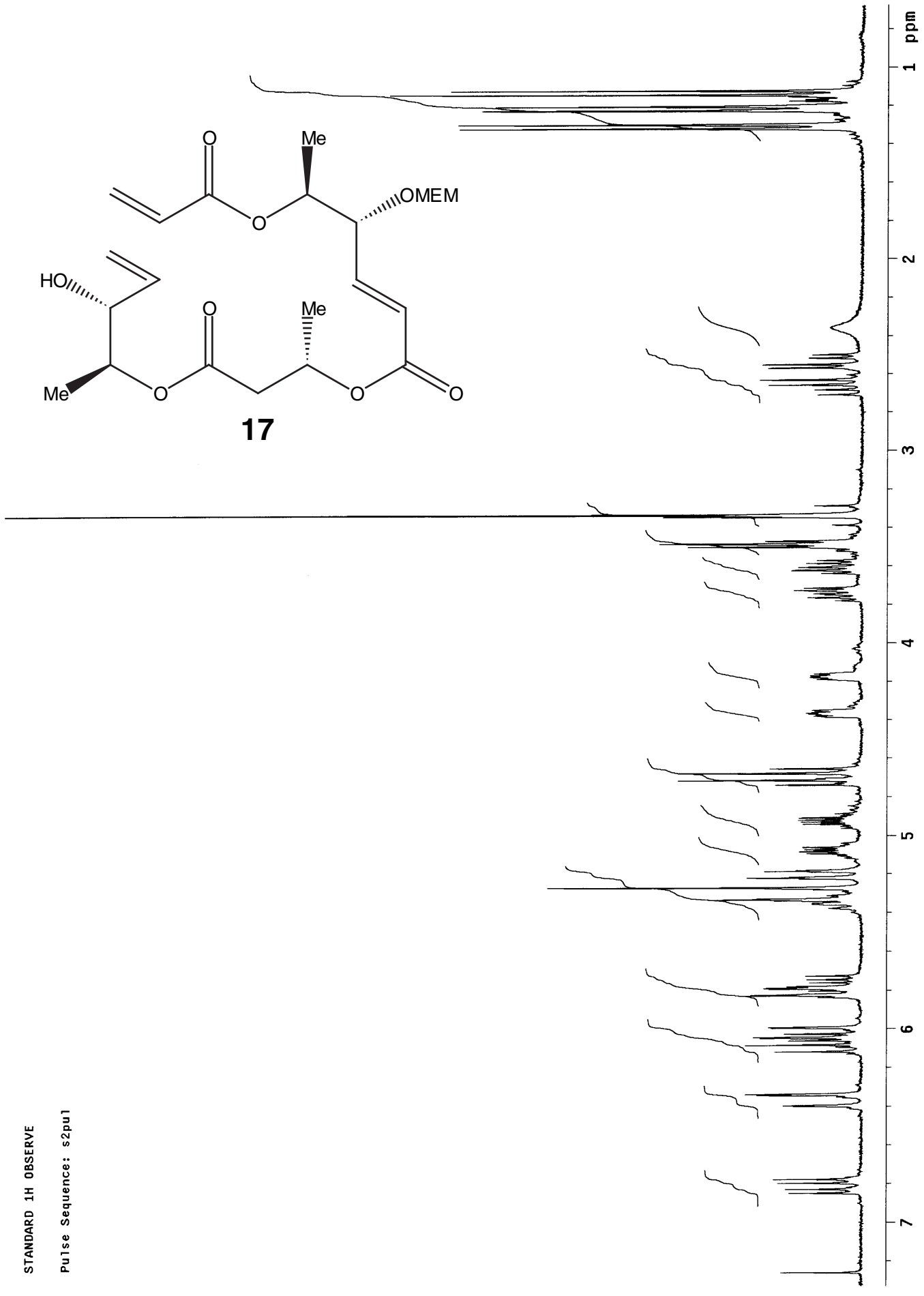



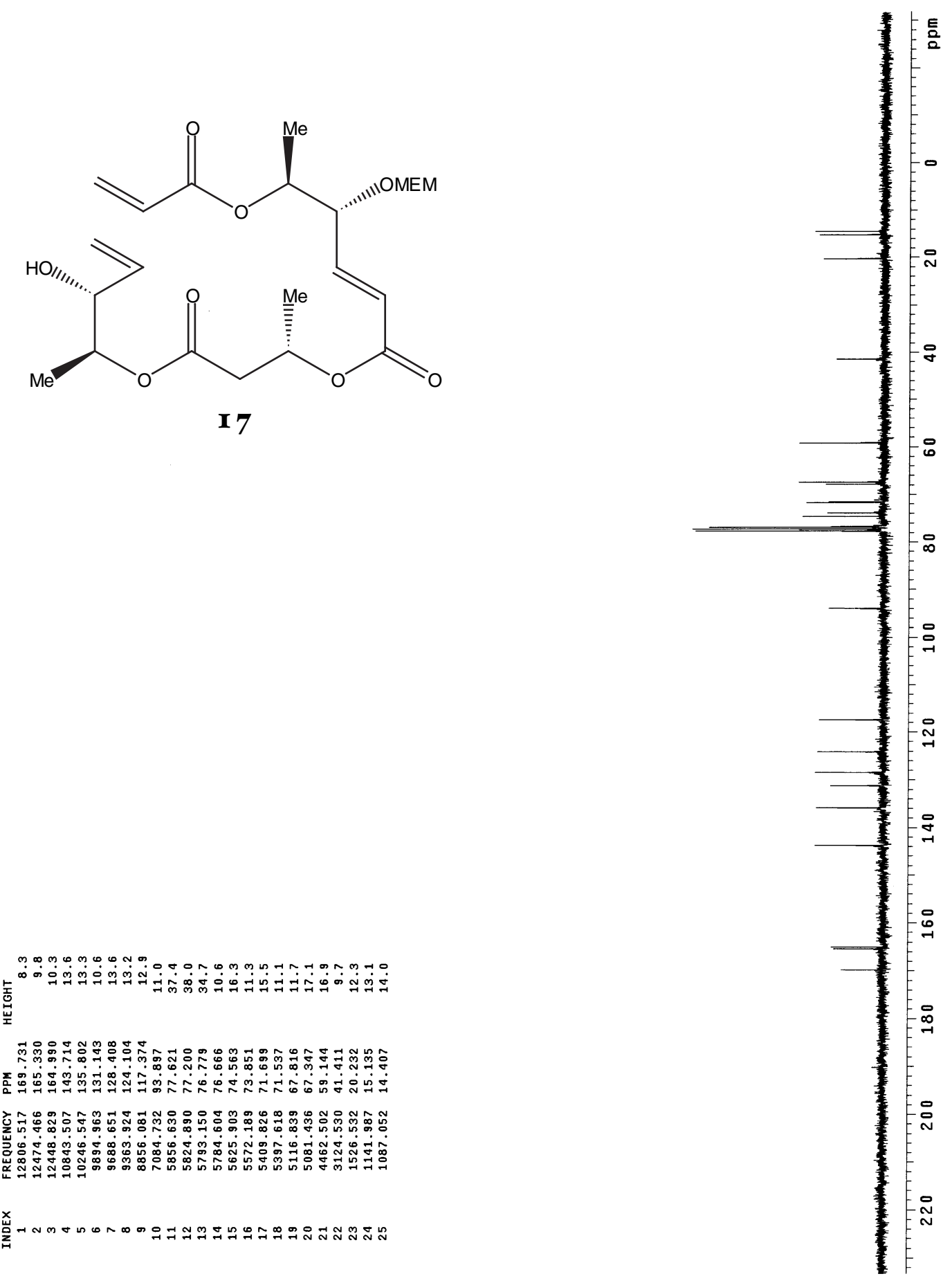


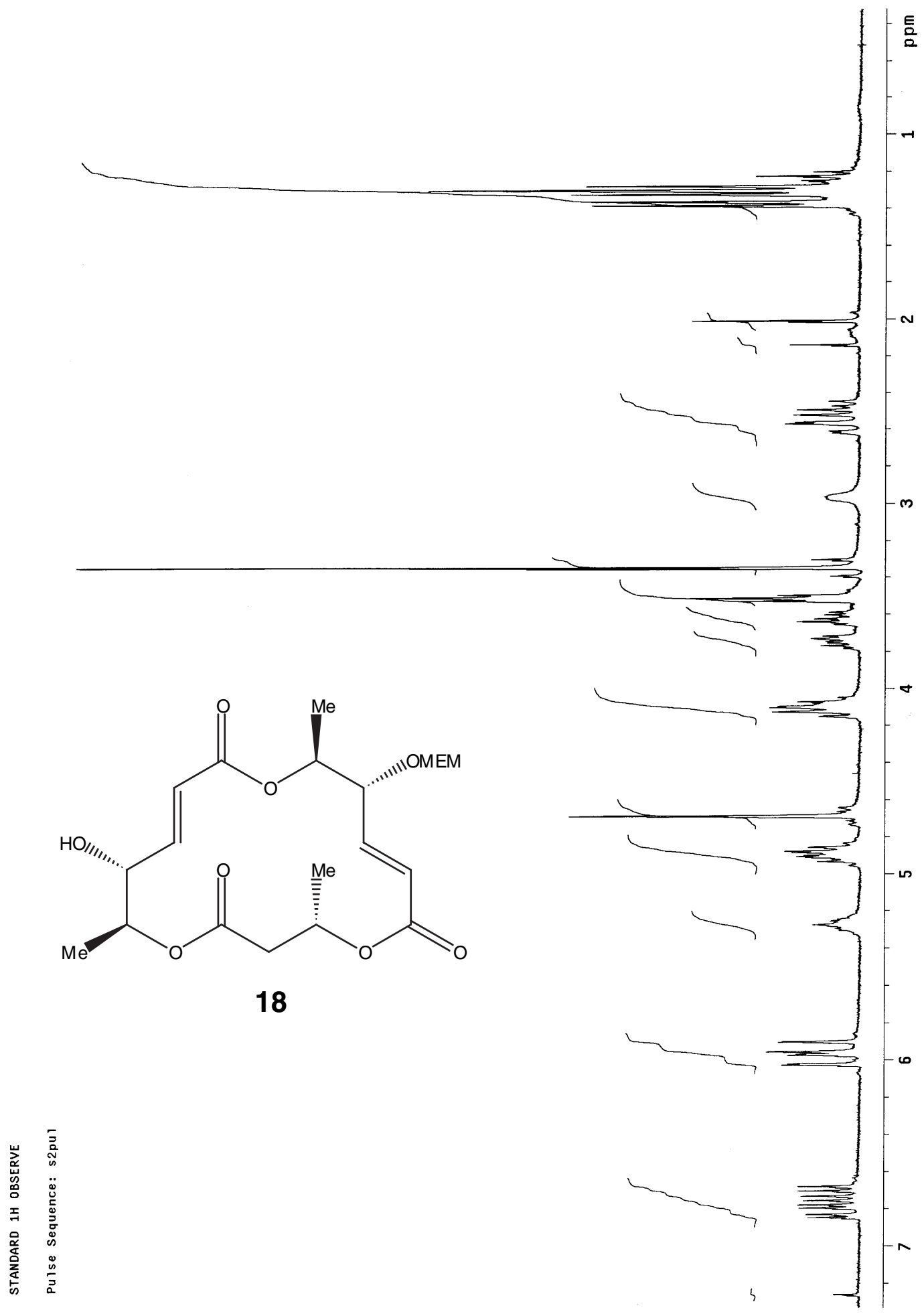



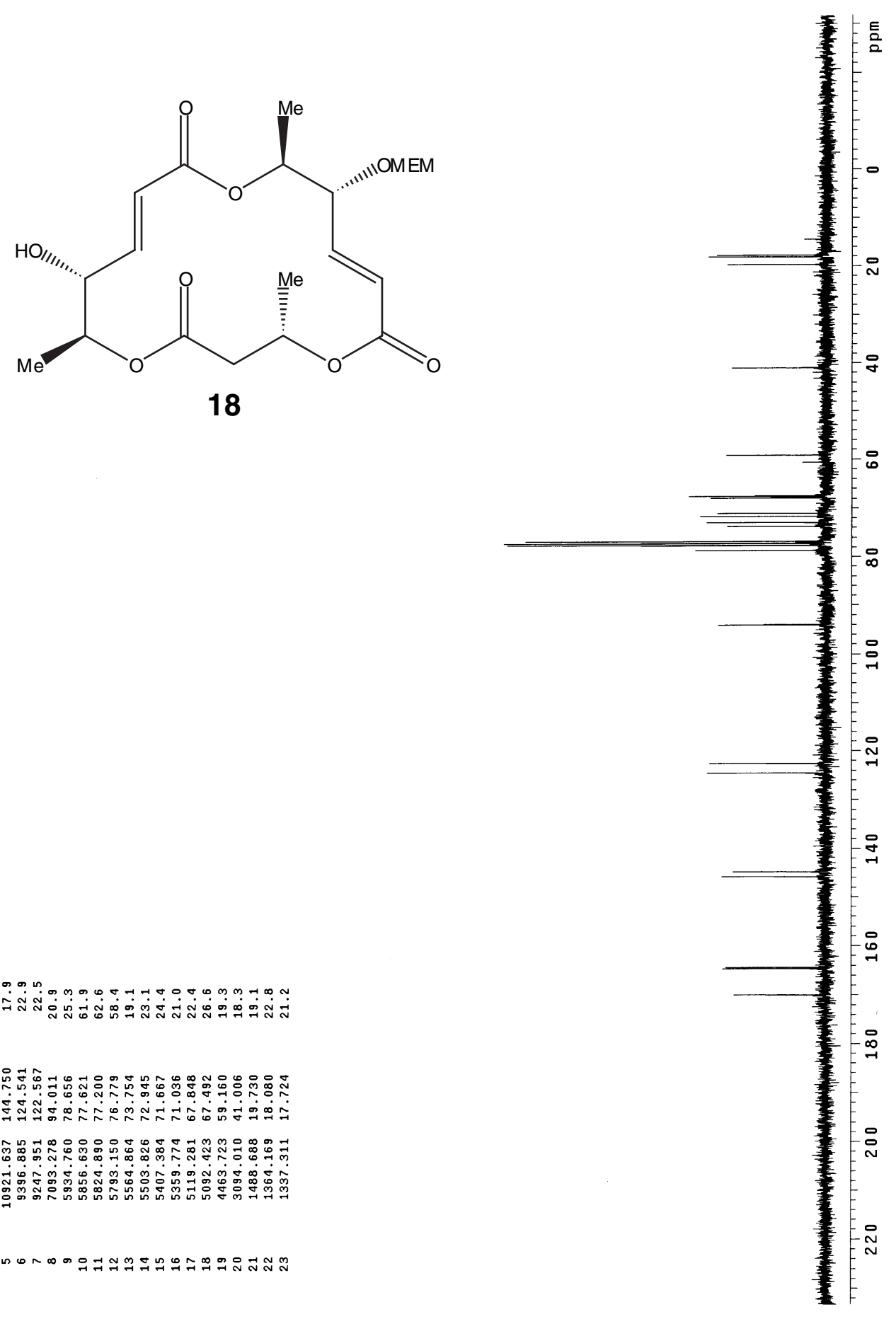


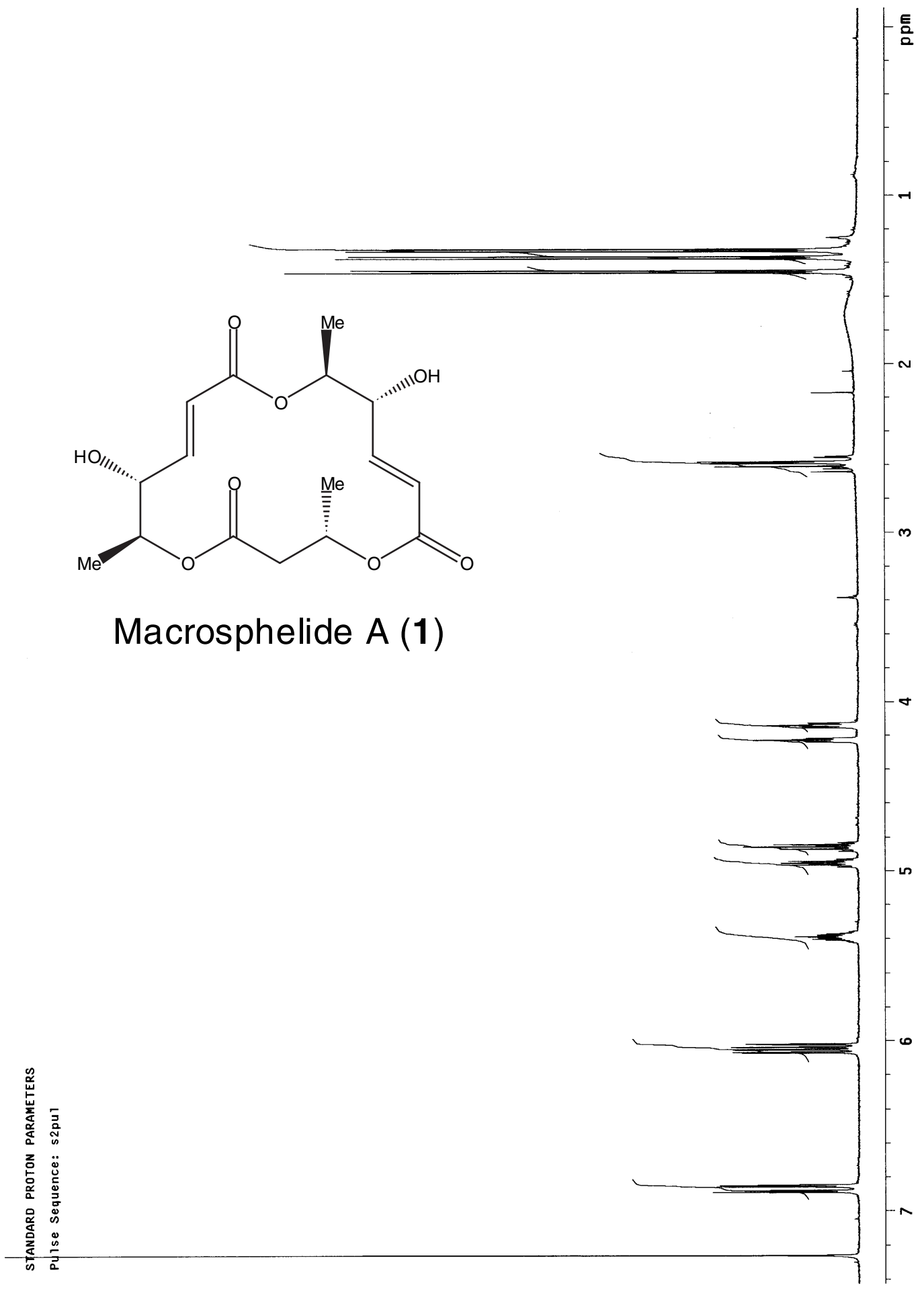



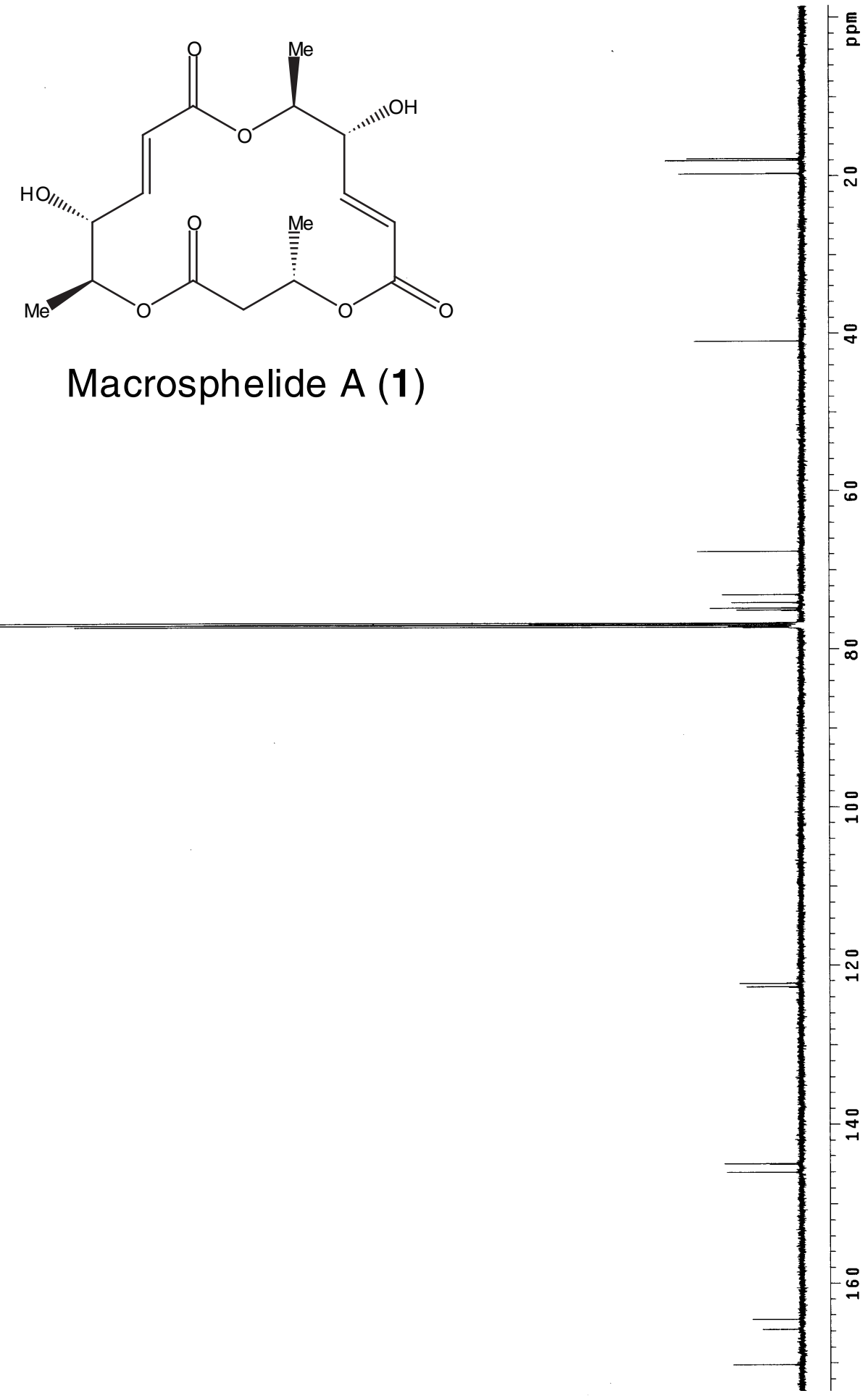


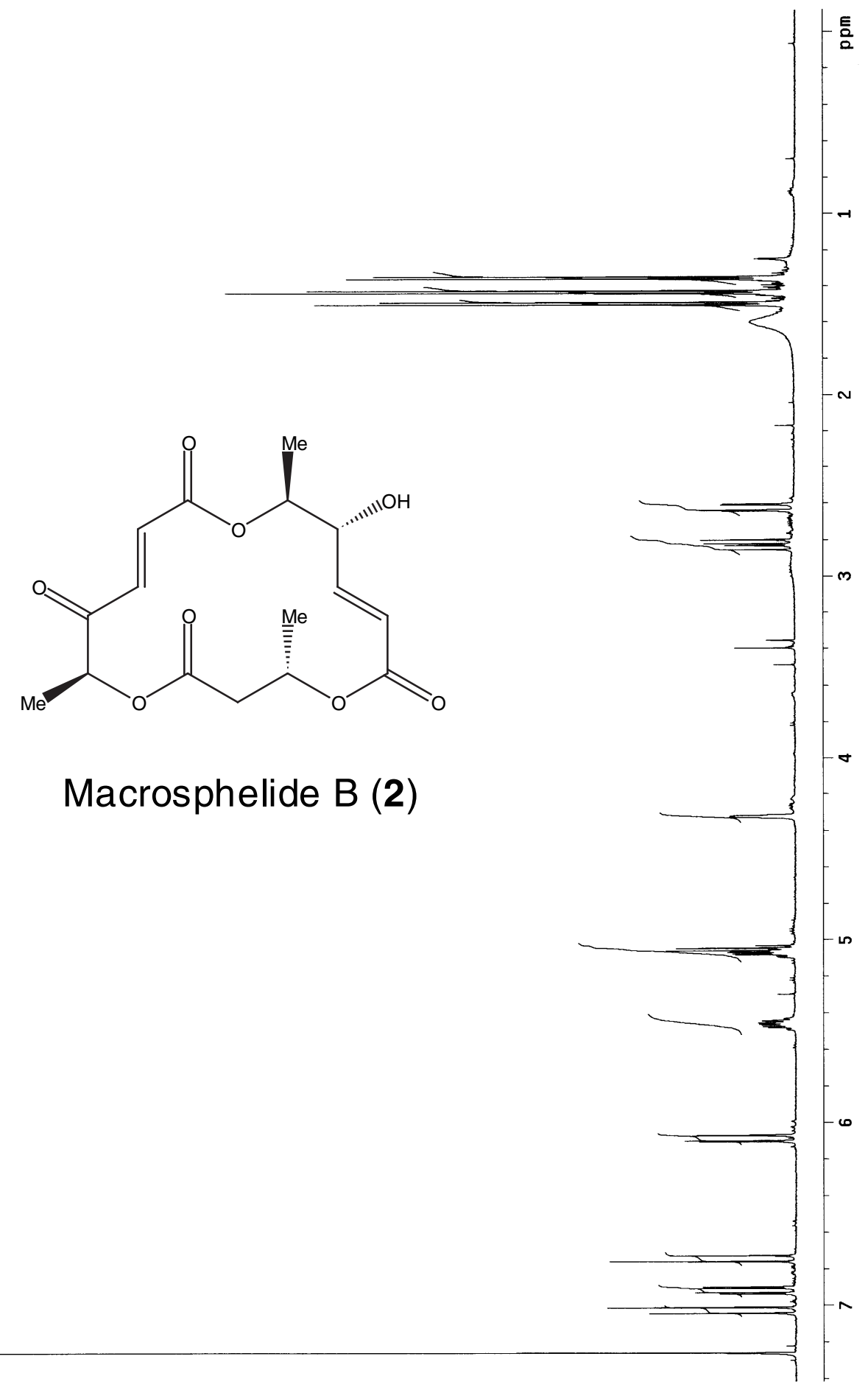




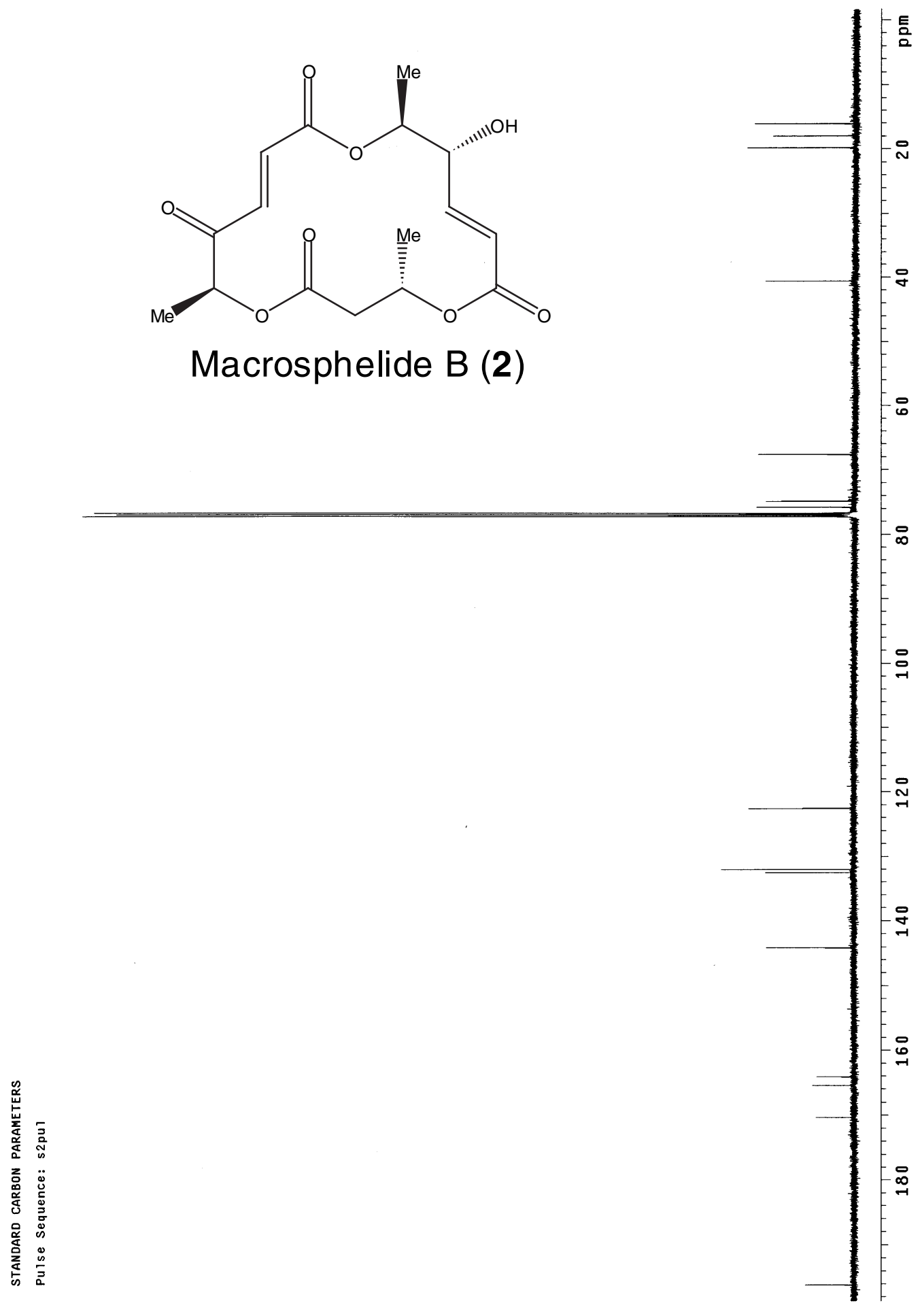

